\title{
Cycle-finite module categories
}

Piotr Malicki, José Antonio de la Peña and Andrzej Skowroński

\begin{abstract}
We describe the structure of module categories of finite dimensional algebras over an algebraically closed field for which the cycles of nonzero nonisomorphisms between indecomposable finite dimensional modules are finite (do not belong to the infinite Jacobson radical of the module category). Moreover, geometric and homological properties of these module categories are exhibited.
\end{abstract}

\section{Introduction}

Throughout the article $K$ denotes a fixed algebraically closed field.

By an algebra we mean an associative finite dimensional $K$-algebra with an identity which we shall assume (without loss of generality) to be basic and connected. For an algebra $A$, by an $A$-module we mean a finite dimensional right $A$-module. We shall denote by $\bmod A$ the category of $A$-modules, by ind $A$ its full subcategory formed by the indecomposable modules, by $\Gamma_{A}$ the Auslander-Reiten quiver of $A$, and by $\tau_{A}$ the Auslander-Reiten translation $D \operatorname{Tr}$ in $\Gamma_{A}$. We shall identify an indecomposable $A$-module with the vertex of $\Gamma_{A}$ corresponding to it. From Drozd's Tame and Wild Theorem [30] (see also [26]) the class of algebras may be divided into two classes. One class consists of the wild algebras whose representation theory comprises the representation theories of all algebras over $K$ (see 68 , Chapter

Piotr Malicki

Faculty of Mathematics and Computer Science, Nicolaus Copernicus University, Chopina 12/18, 87-100 Toruń, Poland, e-mail:pmalicki@mat.uni.torun.pl

José A. de la Peña

Centro de Investigación en Mathemáticas (CIMAT), Guanajuato, México, e-mail: japecimat.mx

Andrzej Skowroński

Faculty of Mathematics and Computer Science, Nicolaus Copernicus University, Chopina 12/18, 87-100 Toruń, Poland, e-mail: skowron@mat.uni.torun.pl 
XIX]). The second class consists of the tame algebras for which the indecomposable modules occur, in each dimension $d$, in a finite number of discrete and a finite number of one-parameter families. Hence, a classification of the finite dimensional modules is only feasible for tame algebras. It has been shown by Crawley-Boevey [26] that, if $A$ is a tame algebra, then, for any dimension $d \geq 1$, all but finitely many isomorphism classes of indecomposable $A$-modules of dimension $d$ are invariant on the action of $\tau_{A}$, and hence, by a result due to Hoshino [35], lie in stable tubes of rank one in $\Gamma_{A}$. The indecomposable modules over tame algebras which do not lie in stable tubes of rank one are called discrete. A distinguished class of tame algebras is formed by the algebras of finite representation type, having only finitely many isomorphism classes of indecomposable modules, for which the representation theory is presently rather well understood (see [13], [20], [21], [22], [24]). On the other hand, the representation theory of arbitrary tame algebras is still only emerging. At present the most accessible seem to be the (tame) algebras of polynomial growth, for which there exists an integer $m$ such that the number of one-parameter families of indecomposable modules is bounded, in each dimension $d$, by $d^{m}$. This class of algebras has been subject of intensive research over the last 30 years.

A prominent role in the representation theory of algebras is played by cycles of modules, or more generally cycles of complexes of modules. Recall that a cycle in a module category $\bmod A$ is a sequence

$$
X_{0} \stackrel{f_{1}}{\longrightarrow} X_{1} \rightarrow \cdots \rightarrow X_{r-1} \stackrel{f_{r}}{\longrightarrow} X_{r}=X_{0}
$$

of nonzero nonisomorphisms in ind $A$, and the cycle is said to be finite if the homomorphisms $f_{1}, \ldots, f_{r}$ do not belong to the infinite Jacobson radical of $\bmod A$. Following Ringel [65] a module in ind $A$ which does not lie on cycle in ind $A$ is called directing. It has been proved independently by Peng and Xio [53] and the third named author [74] that the Auslander-Reiten quiver $\Gamma_{A}$ of an arbitrary algebra $A$ contains at most finitely many $\tau_{A}$-orbits containing directing modules. Hence, in order to obtain information on nondirecting indecomposable modules of a module category, we may study properties of cycles in $\bmod A$ containing these modules. We also note that, by a result of Ringel [65] the support algebras of directing modules are tilted algebras. Following [5] an algebra $A$ is said to be cycle-finite if all cycles in $\bmod A$ are finite. It has been proved by the third named author in [76] that every cycle-finite algebra $A$ is of polynomial growth and the support algebras of the one-parametric families of indecomposable $A$-modules are tame concealed algebras (preprojective tilts of the path algebras of Euclidean quivers) and Ringel's tubular algebras, which are distinguished classes of cycle-finite algebras. The class of cycle-finite algebras is wide and contains the algebras of finite representation type, the tame tilted algebras [37], the tame double tilted algebras [62], the tame generalized double tilted algebras [63], the tubular algebras [65], the iterated tubular algebras [61], the tame quasi-tilted algebras [40], [78], the tame coil and multicoil algebras [5], [6], [7], the tame generalized multicoil algebras [51], and the strongly simply connected algebras of polynomial growth [77]. It has been also proved in [2], [3], [4] that the class of algebras $A$ for which the derived category $D^{b}(\bmod A)$ of 
bounded complexes of $A$-modules is cycle-finite coincides with the class of piecewise hereditary algebras of Dynkin, Euclidean, and tubular type, and consequently these algebras are also cycle-finite. Moreover, frequently an algebra $A$ admits a Galois covering $R \rightarrow R / G=A$ where $R$ is a cycle-finite locally bounded category and $G$ is an admissible group of automorphisms of $R$, which allows to reduce the representation theory of $A$ to the representation theory of cycle-finite algebras being finite convex subcategories of $R$. For example, every selfinjective algebra $A$ of polynomial growth admits a canonical standard form $\bar{A}$ (geometric socle deformation of $A$ ) such that $\bar{A}$ has a Galois covering $R \rightarrow R / G=\bar{A}$, where $R$ is a cycle-finite selfinjective locally bounded category and $G$ is an admissible infinite cyclic group of automorphisms of $R$, the Auslander-Reiten quiver $\Gamma_{\bar{A}}$ of $\bar{A}$ is the orbit quiver $\Gamma_{R} / G$ of $\Gamma_{R}$, and the stable Auslander-Reiten quivers of $A$ and $\bar{A}$ are isomorphic (see [69], [80] for details). We also mention that, by the main result of [59], every algebra $A$ which admits a cycle-finite Galois covering $R \rightarrow R / G=A$ with $G$ torsion-free is tame.

One of the objectives of this article is to describe the structure of the category ind $A$ of an arbitrary cycle-finite algebra $A$, by showing that it can be covered by the categories of indecomposable modules of tame generalized multicoil algebras and tame generalized double tilted algebras. Here, a crucial role will be played by description of support algebras of cyclic components of the Auslander-Reiten quivers of cycle-finite algebras. The second objective of the article is to exhibit geometric and homological properties of indecomposable modules over cycle-finite algebras. We are interested in the class of coherent cycle-finite algebras for which all cyclic components of the Auslander-Reiten quivers are coherent (see Section 2 for definition). Every coherent cycle-finite algebra $A$ is triangular, and hence the (geometric) Tits quadratic form $q_{A}$ and the (homological) Euler form $\chi_{A}$ of $A$ are well defined. For a vector $\mathbf{d}$ in the Grothendieck group $K_{0}(A)$ of $A$ with nonnegative coordinates, we denote by $\bmod _{A}(\mathbf{d})$ the affine variety of $A$-modules of dimension vector $\mathbf{d}$ and by $G(\mathbf{d})$ the corresponding product of general linear groups acting on $\bmod _{A}(\mathbf{d})$ in such a way that the $G(\mathbf{d})$-orbits in $\bmod _{A}(\mathbf{d})$ correspond to the isomorphism classes of $A$-modules of dimension vector $\mathbf{d}$. The third main aim of the article is to establish a common bound on the numbers of discrete indecomposable modules in each dimension vector over cycle-finite algebras, generalizing results proved in [84] for strongly simply connected algebras of polynomial growth.

For basic background from the representation theory of algebras we refer to the books [1], [11], [65], [67], [68], [82].

\section{Preliminaries}

In this section we recall some concepts and results from the representation theory of algebras important for further considerations.

Let $A$ be an algebra (which by our assumption is basic and connected). Then there is an isomorphism $A \cong K Q / I$ of $K$-algebras, where $K Q$ is the path algebra of the Gabriel quiver $Q=Q_{A}$ of $A$ and $I$ is an admissible ideal of $K Q$. Equivalently, 
$A=K Q / I$ may be considered as a $K$-category whose class of objects is the set $Q_{0}$ of vertices of $Q$, and the set of morphisms $A(x, y)$ from $x$ to $y$ is the quotient of $K$-space $K Q(x, y)$, formed by the $K$-linear combinations of paths in $Q$ from $x$ to $y$, by the subspace $I(x, y)=K Q(x, y) \cap I$. We shall identify an algebra $A$ with its $K$ category. Moreover, the module category $\bmod A$ may be identified with the category $\operatorname{rep}_{K}(Q, I)$ of finite dimensional $K$-linear representations of the bound quiver $(Q, I)$. An algebra $A$ with $Q_{A}$ acyclic (without oriented cycles) is said to be triangular. A full subcategory $C$ of $A$ is said to be convex if any path in $Q_{A}$ with source and target in $Q_{C}$ lies entirely in $Q_{C}$. Recall also that the Jacobson radical $\operatorname{rad}(\bmod A)$ of the module category $\bmod A$ is the ideal of $\bmod A$ generated by all noninvertible morphisms in ind $A$. Then the infinite radical $\operatorname{rad}^{\infty}(\bmod A)$ of $\bmod A$ is the intersection of all powers $\operatorname{rad}^{i}(\bmod A), i \geq 1, \operatorname{of} \operatorname{rad}(\bmod A)$. A path of length $t \geq 1 \mathrm{in} \bmod A$ is a sequence of nonzero nonisomorphisms

$$
M_{0} \stackrel{f_{1}}{\longrightarrow} M_{1} \rightarrow \cdots \rightarrow M_{t-1} \stackrel{f_{t}}{\longrightarrow} M_{t}
$$

and modules $M_{0}, M_{1}, \ldots, M_{t}$ in ind $A$. Such a path is said to be finite if $f_{1}, \ldots, f_{t}$ do not belong to $\operatorname{rad}^{\infty}(\bmod A)$, and otherwise infinite. Moreover, if $M_{0} \cong M_{t}$ then the path is called a cycle of length $t$. A module $M$ from ind $A$ is called directing if it does not lie on a cycle in $\bmod A$. For a $\operatorname{module} M$ in $\bmod A$, we denote by $\operatorname{dim} M$ its dimension vector $\left(\operatorname{dim}_{K} M(i)\right)_{i \in Q_{0}}$. The support $\operatorname{supp} M$ of a module $M$ in $\bmod A$ is the full subcategory of $A$ given by all vertices $i$ of $Q_{A}$ such that $M(i) \neq 0$. A module $M$ in $\bmod A$ with $\operatorname{supp} M=A$ is said to be sincere. Recall also that the Grothendieck group $K_{0}(A)=K_{0}(\bmod A)$ is isomorphic to $\mathbb{Z}^{Q_{0}}$.

Let $A$ be an algebra and $K[x]$ the polynomial algebra in one variable $x$. Following [30] $A$ is said to be tame if, for any dimension $d$, there exists a finite number of $K[x]-A$-bimodules $M_{i}, 1 \leq i \leq n_{d}$, which are finitely generated and free as left $K[x]$ modules, and all but a finite number of isoclasses of indecomposable $A$-modules of dimension $d$ are of the form $K[x] /(x-\lambda) \otimes_{K[x]} M_{i}$ for some $\lambda \in K$ and some $i \in\left\{1, \ldots, n_{d}\right\}$. Let $\mu_{A}(d)$ be the least number of $K[x]-A$-bimodules $M_{i}$ satisfying the above condition for $d$. Then $A$ is said to be of polynomial growth (respectively, domestic) if there exists a positive integer $m$ such that $\mu_{A}(d) \leq d^{m}$ (respectively, $\mu_{A}(d) \leq m$ ) for any $d \geq 1$ (see [27], [70]). Recall that from the validity of the second Brauer-Thrall conjecture we know that $A$ is representation-finite if and only if $\mu_{A}(d)=0$ for any $d \geq 1$.

The Tits form of a triangular algebra $A=K Q / I$ is the integral quadratic form $q_{A}: \mathbb{Z}^{Q_{0}} \rightarrow \mathbb{Z}$, defined, for $\mathbf{x}=\left(x_{i}\right)_{i \in Q_{0}} \in \mathbb{Z}^{Q_{0}}$, by

$$
q_{A}(\mathbf{x})=\sum_{i \in Q_{0}} x_{i}^{2}-\sum_{(i \rightarrow j) \in Q_{1}} x_{i} x_{j}+\sum_{i, j \in Q_{0}} r(i, j) x_{i} x_{j}
$$

where $Q_{1}$ is the set of arrows in $Q$ and $r(i, j)$ is the cardinality of $L \cap I(i, j)$, for a minimal set of generators $L \subset \bigcup_{i, j \in Q_{0}} I(i, j)$ of the ideal $I$ (see [18]). Moreover, the Euler form of $A$ is the integral quadratic form $\chi_{A}: \mathbb{Z}^{Q_{0}} \rightarrow \mathbb{Z}$ defined in [65, 2.4] such that for any module $M$ in $\bmod A$, we have 


$$
\chi_{A}(\operatorname{dim} M)=\sum_{i=0}^{\infty}(-1)^{i} \operatorname{dim}_{K} \operatorname{Ext}_{A}^{i}(M, M) .
$$

Observe that $A$ is of finite global dimension, because $A$ is triangular. It is also known that if $\operatorname{gl} \operatorname{dim} A \leq 2$ then $q_{A}=\chi_{A}$ (see [18]). Finally, it is known (see [54]) that, if $A$ is tame, then $q_{A}$ is weakly nonnegative, that is, $q_{A}(\mathbf{x}) \geq 0$ for all $\mathbf{x} \in \mathbb{N} Q_{0}$. Unfortunately, the reverse implication is not true in general. However, it has been proved recently in [25] that a strongly simply connected algebra $A$ is tame if and only if the Tits form $q_{A}$ is weakly nonnegative. Recall also that a triangular algebra $A$ is called strongly simply connected [71] if the first Hochschild cohomology $H^{1}(C, C)$ of every convex subcategory $C$ of $A$ vanishes.

We need also special types of components of the Auslander-Reiten quivers of algebras.

Recall from [28], [65] that a translation quiver $\Gamma$ is called a tube if it contains a cyclical path and if its underlying topological space is homeomorphic to $S^{1} \times \mathbb{R}^{+}$, where $S^{1}$ is the unit circle and $\mathbb{R}^{+}$is the nonnegative real line. A tube has only two types of arrows: arrows pointing to infinity and arrows pointing to the mouth. Tubes containing neither projective vertices nor injective vertices are called stable, and are as follows. For the infinite quiver

$$
\mathbb{A}_{\infty}: 0 \rightarrow 1 \rightarrow 2 \rightarrow \cdots
$$

the translation quiver $\mathbb{Z} \mathbb{A}_{\infty}$ is of the form

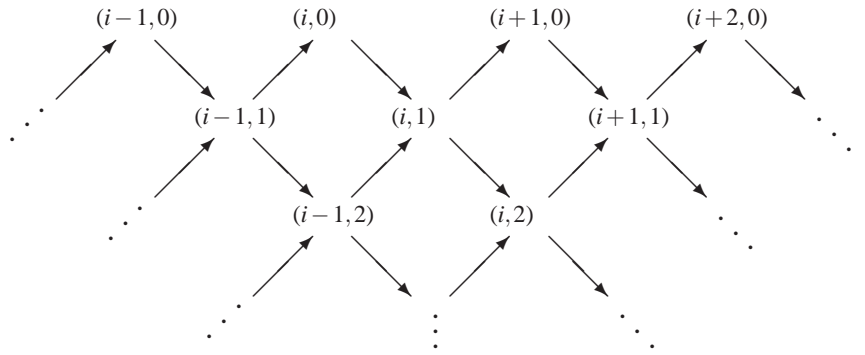

with the translation $\tau$ given by $\tau(i, j)=(i-1, j)$ for $i \in \mathbb{Z}, j \in \mathbb{N}$. For each $r \geq 1$, denote by $\mathbb{Z} \mathbb{A}_{\infty} /\left(\tau^{r}\right)$ the translation quiver $\Gamma$ obtained from $\mathbb{Z} \mathbb{A}_{\infty}$ by identifying each vertex $(i, j)$ of $\mathbb{Z} \mathbb{A}_{\infty}$ with the vertex $\tau^{r}(i, j)$ and each arrow $x \rightarrow y$ in $\mathbb{Z} \mathbb{A}_{\infty}$ with the arrow $\tau^{r} x \rightarrow \tau^{r} y$. The translation quiver $\mathbb{Z} \mathbb{A}_{\infty} /\left(\tau^{r}\right)$ is called the stable tube of rank $r$. The $\tau$-orbit of a stable tube $\Gamma$ formed by all vertices having exactly one immediate predecessor (equivalently, successor) is called the mouth of $\Gamma$. A tube $\Gamma$ without injective vertices (respectively, without projective vertices) is called a ray tube (respectively, coray tube).

Let $A$ be an algebra. A component $\mathscr{C}$ of $\Gamma_{A}$ is called regular if $\mathscr{C}$ contains neither a projective module nor an injective module, and semiregular if $\mathscr{C}$ does not contain both a projective and an injective module. It has been shown in [41] and [87] that a regular component $\mathscr{C}$ of $\Gamma_{A}$ contains an oriented cycle if and only if $\mathscr{C}$ is a stable tube. Moreover, Liu proved in [42] that a semiregular component $\mathscr{C}$ of $\Gamma_{A}$ contains 
an oriented cycle if and only if $\mathscr{C}$ is a ray or coray tube. A component $\mathscr{P}$ of $\Gamma_{A}$ is called postprojective if $\mathscr{P}$ is acyclic and every module in $\mathscr{P}$ lies in the $\tau_{A}$-orbit of a projective module. Dually, a component $\mathscr{Q}$ of $\Gamma_{A}$ is called preinjective if $\mathscr{Q}$ is acyclic and every module in $\mathscr{Q}$ lies in the $\tau_{A}$-orbit of an injective module. A component $\Gamma$ of $\Gamma_{A}$ is said to be coherent if the following two conditions are satisfied:

(C1) For each projective module $P$ in $\Gamma$ there is an infinite sectional path $P=X_{1} \rightarrow X_{2} \rightarrow \cdots \rightarrow X_{i} \rightarrow X_{i+1} \rightarrow X_{i+2} \rightarrow \cdots$ (that is, $X_{i} \neq \tau_{A} X_{i+2}$ for any $i \geq 1)$ in $\Gamma$.

(C2) For each injective module $I$ in $\Gamma$ there is an infinite sectional path $\cdots \rightarrow$ $Y_{j+2} \rightarrow Y_{j+1} \rightarrow Y_{j} \rightarrow \cdots \rightarrow Y_{2} \rightarrow Y_{1}=I$ (that is, $Y_{j+2} \neq \tau_{A} Y_{j}$ for any $j \geq 1$ ) in $\Gamma$. Further, a component $\Gamma$ of $\Gamma_{A}$ is said to be almost cyclic if all but finitely many modules of $\Gamma$ lie on oriented cycles in $\Gamma_{A}$, so contained entirely in $\Gamma$. We note that the stable tubes, ray tubes and coray tubes of $\Gamma_{A}$ are special types of almost cyclic coherent components. In general, it has been proved in [50] that a component $\Gamma$ of $\Gamma_{A}$ is almost cyclic and coherent if and only if $\Gamma$ is a generalized multicoil, which can be obtained from a family of stable tubes by a sequence of admissible operations (see Section 4 for algebras having such components). A component $\Gamma$ of $\Gamma_{A}$ is said to be almost acyclic if all but finitely many modules of $\Gamma$ are acyclic (do not lie on oriented cycles in $\Gamma_{A}$, hence in $\Gamma$ ), and acyclic if all modules of $\Gamma$ are acyclic. Finally, following [73] a component $\mathscr{C}$ of $\Gamma_{A}$ is said to be generalized standard if $\operatorname{rad}_{A}^{\infty}(X, Y)=0$ for all modules $X$ and $Y$ from $\mathscr{C}$. It has been proved in [73] that every generalized standard component $\mathscr{C}$ of $\Gamma_{A}$ is almost periodic, that is, all but finitely many $\tau_{A}$-orbits in $\mathscr{C}$ are periodic. Clearly, the postprojective and preinjective components are acyclic, and the Auslander-Reiten quivers of representation-finite algebras are almost acyclic. Moreover, these components are generalized standard (see [74]). General results on almost acyclic components and related algebras have been proved by Reiten and third named author in [62], [63] (see Section 5). For a component $\mathscr{C}$ of $\Gamma_{A}$, we denote by $\operatorname{ann}_{A}(\mathscr{C})$ the annihilator of $\mathscr{C}$ in $A$, that is, the intersection of the annihilators $\operatorname{ann}_{A}(X)=\{a \in A \mid X a=0\}$ of all modules $X$ in $\mathscr{C}$. We note that $\mathscr{C}$ is a component of $\Gamma_{A} / \operatorname{ann}_{A}(\mathscr{C})$. Moreover, if $\operatorname{ann}_{A}(\mathscr{C})=0, \mathscr{C}$ is said to be a faithful component of $\Gamma_{A}$. By the support of a subquiver $\Gamma$ of $\Gamma_{A}$ we mean the full subcategory $\operatorname{supp} \Gamma$ of $A$ given by the supports supp $M$ of all modules $M$ in $\Gamma$, and, if $\operatorname{supp} \Gamma=A$ then $\Gamma$ is said to be sincere. We note that a faithful component $\mathscr{C}$ of $\Gamma_{A}$ is sincere.

\section{Semiregular components of cycle-finite algebras}

In this section we recall the shapes of the Auslander-Reiten quivers of representationinfinite tilted algebras of Euclidean type and tubular algebras, as well as results from [76] on semiregular components of the Auslander-Reiten quivers of cycle-finite algebras, important for further considerations.

By a tame concealed algebra we mean a tilted algebra $C=\operatorname{End}_{H}(T)$, where $H$ is the path algebra $K \Delta$ of a quiver $\Delta$ of Euclidean type (the underlying graph $\bar{\Delta}$ of 
$\Delta$ of type $\widetilde{\mathbb{A}}_{m}(m \geq 1), \widetilde{\mathbb{D}}_{n}(n \geq 4)$, or $\left.\widetilde{\mathbb{E}}_{p}(6 \leq p \leq 8)\right)$ and $T$ is a (multiplicity-free) postprojective tilting $H$-modules. The tame concealed algebras have been described by quivers and relations by Bongartz [19] and Happel-Vossieck [34]. Recall also that the Auslander-Reiten quiver $\Gamma_{C}$ of a tame concealed algebra $C$ is of the form

$$
\Gamma_{C}=\mathscr{P}^{C} \cup \mathscr{T}^{C} \cup \mathscr{Q}^{C},
$$

where $\mathscr{P}^{C}$ is a postprojective component containing all indecomposable projective $C$-modules, $\mathscr{Q}^{C}$ is a preinjective component containing all indecomposable injective $C$-modules, and $\mathscr{T}^{C}$ is a $\mathbb{P}_{1}(K)$-family $\mathscr{T}_{\lambda}^{C}, \lambda \in \mathbb{P}_{1}(K)$, of pairwise orthogonal generalized standard stable tubes, all but a finite number of them of rank one. The ordering from the left to right indicates that there are nonzero homomorphisms only from any of these classes to itself and to the classes to its right. We refer to 65 , Chapter 4] and [67] for more details on the module categories of tame concealed algebras.

Recall also that, if $B$ is a representation-infinite tilted algebra of Euclidean type $\Delta$, then one of the following holds:

(a) $B$ is a domestic tubular extension of a tame concealed algebra $C$ and

$$
\Gamma_{B}=\mathscr{P}^{B} \cup \mathscr{T}^{B} \cup \mathscr{Q}^{B},
$$

where $\mathscr{P}^{B}=\mathscr{P}^{C}$ is the postprojective component of $\Gamma_{C}, \mathscr{T}^{B}$ is a $\mathbb{P}_{1}(K)$-family $\mathscr{T}_{\lambda}^{B}$, $\lambda \in \mathbb{P}_{1}(K)$, of pairwise orthogonal generalized standard ray tubes, obtained from the $\mathbb{P}_{1}(K)$-family $\mathscr{T}^{C}$ of stable tubes of $\Gamma_{C}$ by ray insertions, and $\mathscr{Q}^{B}$ is a preinjective component containing all indecomposable injective $B$-modules and a section of type $\Delta$

(b) $B$ is a domestic tubular coextension of a tame concealed algebra $C$ and

$$
\Gamma_{B}=\mathscr{P}^{B} \cup \mathscr{T}^{B} \cup \mathscr{Q}^{B},
$$

where $\mathscr{P}^{B}$ is the postprojective component containing all indecomposable projective $B$-modules and a section of type $\Delta, \mathscr{T}^{B}$ is a $\mathbb{P}_{1}(K)$-family $\mathscr{T}_{\lambda}^{B}, \lambda \in \mathbb{P}_{1}(K)$, of pairwise orthogonal generalized standard coray tubes, obtained from the $\mathbb{P}_{1}(K)$ family $\mathscr{T}^{C}$ of stable tubes of $\Gamma_{C}$ by coray insertions, and $\mathscr{Q}^{B}=\mathscr{Q}^{C}$ is the preinjective component of $\Gamma_{C}$. We refer to [65, Chapter 4] and [68, Chapters XV-XVII] for more details on the module categories of representation-infinite tilted algebras of Euclidean type.

By a tubular algebra we mean a tubular extension (equivalently tubular coextension) of a tame concealed algebra of tubular type $(2,2,2,2),(3,3,3),(2,4,4)$, or $(2,3,6)$, as defined by Ringel in [65, Chapter 5]. Recall that a tubular algebra $B$ admits two different tame concealed convex subcategories $C_{0}$ and $C_{\infty}$ such that $B$ is a tubular extension of $C_{0}$, and a tubular coextension of $C_{\infty}$, and the Auslander-Reiten quiver $\Gamma_{B}$ of $B$ is of the form 


$$
\Gamma_{B}=\mathscr{P}_{0}^{B} \cup \mathscr{T}_{0}^{B} \cup\left(\bigcup_{q \in \mathbb{Q}^{+}} \mathscr{T}_{q}^{B}\right) \cup \mathscr{T}_{\infty}^{B} \cup \mathscr{Q}_{\infty}^{B},
$$

where $\mathscr{P}_{0}^{B}=\mathscr{P}^{C_{0}}$ is the postprojective component of $\Gamma_{C_{0}}, \mathscr{T}_{0}^{B}$ is a $\mathbb{P}_{1}(K)$-family of pairwise orthogonal generalized standard ray tubes, obtained from the $\mathbb{P}_{1}(K)$ family $\mathscr{T}^{C_{0}}$ of stable tubes of $\Gamma_{C_{0}}$ by ray insertions, $\mathscr{Q}_{\infty}^{B}=\mathscr{Q}^{C_{\infty}}$ is the preinjective component of $\Gamma_{C_{\infty}}, \mathscr{T}_{\infty}^{B}$ is a $\mathbb{P}_{1}(K)$-family of pairwise orthogonal generalized standard coray tubes, obtained from the $\mathbb{P}_{1}(K)$-family $\mathscr{T}^{C_{\infty}}$ of stable tubes of $\Gamma_{C_{\infty}}$ by coray insertions, and, for each $q \in \mathbb{Q}^{+}$(the set of positive rational numbers) $\mathscr{T}_{q}^{B}$ is a $\mathbb{P}_{1}(K)$-family of pairwise orthogonal generalized standard stable tubes. We refer to [65, Chapter 5] for more details on the module categories of tubular algebras.

The following characterization of tame concealed and tubular algebras has been established in [76, Theorem 4.1].

Theorem 3.1. Let A be an algebra. The following statements are equivalent:

(i) $A$ is cycle-finite and $\Gamma_{A}$ admits a sincere stable tube.

(ii) A is either tame concealed or tubular.

Moreover, we have also the following consequence [76, Theorem 4.3] of the above theorem, the tameness of cycle-finite algebras [5, Proposition 1.4], and a result of Crawley-Boevey [26, Corollary E].

Theorem 3.2. Let A be a cycle-finite algebra. Then A is of polynomial growth.

An algebra $A$ is said to be minimal representation-infinite if $A$ is representationinfinite but every proper convex subcategory of $A$ is representation-finite. Then we have the following characterization of minimal representation-infinite cycle-finite algebras established in [76, Corollary 4.4].

Theorem 3.3. Let $A$ be an algebra. The following statements are equivalent:

(i) $A$ is minimal representation-infinite and cycle-finite.

(ii) A is a tame concealed algebra.

We also have the following characterization of domestic cycle-finite algebras established in [76, Theorem 5.1].

Theorem 3.4. Let A be a cycle-finite algebra. The following statements are equivalent:

(i) A is domestic.

(ii) A does not contain a tubular algebra as a convex subcategory.

(iii) All but finitely many components of $\Gamma_{A}$ are stable tubes of rank one.

In general, we have the following information on the Auslander-Reiten quiver of a cycle-finite algebra, which is a consequence of results proved in [74], [76], and results on the shapes of regular components from [41], [87]. 
Theorem 3.5. Let A be a cycle-finite algebra. Then every regular component of $\Gamma_{A}$ is a generalized standard stable tube. In particular, all but finitely many components of $\Gamma_{A}$ are stable tubes.

Finally, we give a complete description of semiregular components of the AuslanderReiten quivers of cycle-finite algebras, established in [76, Proposition 3.3].

Theorem 3.6. Let $A$ be a cycle-finite algebra and $\mathscr{C}$ be a semiregular component of $\Gamma_{A}$ containing a projective module. Then $B=\operatorname{supp} \mathscr{C}$ is a convex subcategory of $A$ and one of the following holds:

(i) $B$ is a domestic tubular coextension of a tame concealed algebra and $\mathscr{C}$ is the postprojective component of $\Gamma_{B}$.

(ii) $B$ is either a domestic tubular extension of a tame concealed algebra or a tubular algebra, and $\mathscr{C}$ is a generalized standard ray tube of $\Gamma_{B}$.

Theorem 3.7. Let $A$ be a cycle-finite algebra and $\mathscr{C}$ be a semiregular component of $\Gamma_{A}$ containing an injective module. Then $B=\operatorname{supp} \mathscr{C}$ is a convex subcategory of $A$ and one of the following holds:

(i) $B$ is a domestic tubular extension of a tame concealed algebra and $\mathscr{C}$ is the preinjective component of $\Gamma_{B}$.

(ii) $B$ is either a domestic tubular coextension of a tame concealed algebra or a tubular algebra, and $\mathscr{C}$ is a generalized standard coray tube of $\Gamma_{B}$.

\section{Tame generalized multicoil algebras}

In this section we introduce and exhibit basic properties of the class of tame generalized multicoil algebras, plying a prominent role in the description of infinite cyclic components of the Auslander-Reiten quivers of cycle-finite algebras. This is the class of tame algebras among the class of all algebras having a separating family of almost cyclic coherent components investigated in [51], [52]. Recall that a family $\mathscr{C}=\left(\mathscr{C}_{i}\right)_{i \in I}$ of components of the Auslander-Reiten quiver $\Gamma_{A}$ of an algebra $A$ is called separating in $\bmod A$ if the modules in ind $A$ split into three disjoint classes $\mathscr{P}^{A}, \mathscr{C}^{A}=\mathscr{C}$ and $\mathscr{Q}^{A}$ such that:

(S1) $\mathscr{C}^{A}$ is a sincere generalized standard family of components;

(S2) $\operatorname{Hom}_{A}\left(\mathscr{Q}^{A}, \mathscr{P}^{A}\right)=0, \operatorname{Hom}_{A}\left(\mathscr{Q}^{A}, \mathscr{C}^{A}\right)=0, \operatorname{Hom}_{A}\left(\mathscr{C}^{A}, \mathscr{P}^{A}\right)=0$;

(S3) any morphism from $\mathscr{P}^{A}$ to $\mathscr{Q}^{A}$ factors through the additive category $\operatorname{add} \mathscr{C}^{A}$ of $\mathscr{C}^{A}$.

We then say that $\mathscr{C}^{A}$ separates $\mathscr{P}^{A}$ from $\mathscr{Q}^{A}$ and write $\Gamma_{A}=\mathscr{P}^{A} \cup \mathscr{C}^{A} \cup \mathscr{Q}^{A}$. We note that then $\mathscr{P}^{A}$ and $\mathscr{Q}^{A}$ are uniquely determined by $\mathscr{C}^{A}$ (see [8, (2.1)] or [65, (3.1)]).

We also recall a characterization of generalized standard stable tubes of an Auslander-Reiten quiver established in [73, Corollary 5.3] (see also [75, Lemma 3.1].

Proposition 4.1. Let $A$ be an algebra and $\Gamma$ be a stable tube of $\Gamma_{A}$. The following statements are equivalent: 
(i) $\Gamma$ is generalized standard.

(ii) The mouth of $\Gamma$ consists of pairwise orthogonal bricks.

(iii) $\operatorname{rad}_{A}^{\infty}(X, X)=0$ for any module $X$ in $\Gamma$.

Recall that a module $X$ in $\bmod A$ is called a brick if $\operatorname{End}_{A}(X) \cong K$.

It has been proved in [50, Theorem A] that a connected component $\Gamma$ of an Auslander-Reiten quiver $\Gamma_{A}$ is almost cyclic and coherent if and only if $\Gamma$ is a generalized multicoil, obtained from a family of stable tubes by a sequence of operations called admissible. We recall the letter and simultaneously define the corresponding enlargements of algebras.

We start with the one-point extensions and one-point coextensions of algebras. Let $A$ be an algebra and $M$ be a module in $\bmod A$. Then the one-point extension of $A$ by $M$ is the matrix algebra

$$
A[M]=\left[\begin{array}{cc}
A & 0 \\
M & K
\end{array}\right]=\left\{\left[\begin{array}{cc}
a & 0 \\
m & \lambda
\end{array}\right] ; \lambda \in K, a \in A, m \in M\right\}
$$

with the usual addition and multiplication. The quiver $Q_{A[M]}$ of $A[M]$ contains the quiver $Q_{A}$ of $A$ as a convex subquiver, and there is an additional (extension) vertex which is a source. The $A[M]$-modules are usually identified with the triples $(V, X, \varphi)$, where $V$ is a $K$-vector space, $X$ an $A$-module and $\varphi: V \rightarrow \operatorname{Hom}_{A}(M, X)$ is a $K$-linear map. An $A[M]$-linear map $(V, X, \varphi) \rightarrow(W, Y, \psi)$ is then identified with a pair $(f, g)$, where $f: V \rightarrow W$ is $K$-linear, $g: X \rightarrow Y$ is $A$-linear and $\psi f=\operatorname{Hom}_{A}(M, g) \varphi$. Dually, one defines also the one-point coextension of $A$ by $M$ as the matrix algebra

$$
[M] A=\left[\begin{array}{cc}
K & 0 \\
D(M) & A
\end{array}\right] .
$$

For $K$ and $r \geq 1$, we denote by $T_{r}(K)$ the $r \times r$-lower triangular matrix algebra

$$
\left[\begin{array}{cccccc}
K & 0 & 0 & \ldots & 0 & 0 \\
K & K & 0 & \ldots & 0 & 0 \\
K & K & K & \ldots & 0 & 0 \\
\vdots & \vdots & \vdots & \ddots & \vdots & \vdots \\
K & K & K & \ldots & K & 0 \\
K & K & K & \ldots & K & K
\end{array}\right]
$$

Given a generalized standard component $\Gamma$ of $\Gamma_{A}$, and an indecomposable module $X$ in $\Gamma$, the support $\mathscr{S}(X)$ of the functor $\left.\operatorname{Hom}_{A}(X,-)\right|_{\Gamma}$ is the $R$-linear category defined as follows [7]. Let $\mathscr{H}_{X}$ denote the full subcategory of $\Gamma$ consisting of the indecomposable modules $M$ in $\Gamma$ such that $\operatorname{Hom}_{A}(X, M) \neq 0$, and $\mathscr{I}_{X}$ denote the ideal of $\mathscr{H}_{X}$ consisting of the morphisms $f: M \rightarrow N$ (with $M, N$ in $\mathscr{H}_{X}$ ) such that $\operatorname{Hom}_{A}(X, f)=0$. We define $\mathscr{S}(X)$ to be the quotient category $\mathscr{H}_{X} / \mathscr{I}_{X}$. Following the above convention, we usually identify the $R$-linear category $\mathscr{S}(X)$ with its quiver. 
From now on let $A$ be an algebra and $\Gamma$ be a family of generalized standard infinite components of $\Gamma_{A}$. For an indecomposable brick $X$ in $\Gamma$, called the pivot, one defines five admissible operations (ad 1)-(ad 5) and their dual $\left(\operatorname{ad~} 1^{*}\right)-\left(\operatorname{ad} 5^{*}\right)$ modifying the translation quiver $\Gamma=(\Gamma, \tau)$ to a new translation quiver $\left(\Gamma^{\prime}, \tau^{\prime}\right)$ and the algebra $A$ to a new algebra $A^{\prime}$, depending on the shape of the support $\mathscr{S}(X)$ (see [50. Section 2] for the figures illustrating the modified translation quivers $\Gamma^{\prime}$ ).

(ad 1) Assume $\mathscr{S}(X)$ consists of an infinite sectional path starting at $X$ :

$$
X=X_{0} \rightarrow X_{1} \rightarrow X_{2} \rightarrow \cdots
$$

In this case, we let $t \geq 1$ be a positive integer, $D=T_{t}(K)$ and $Y_{1}, Y_{2}, \ldots, Y_{t}$ denote the indecomposable injective $D$-modules with $Y=Y_{1}$ the unique indecomposable projective-injective $D$-module. We define the modified algebra $A^{\prime}$ of $A$ to be the one-point extension

$$
A^{\prime}=(A \times D)[X \oplus Y]
$$

and the modified translation quiver $\Gamma^{\prime}$ of $\Gamma$ to be obtained by inserting in $\Gamma$ the rectangle consisting of the modules $Z_{i j}=\left(K, X_{i} \oplus Y_{j},\left[\begin{array}{l}1 \\ 1\end{array}\right]\right)$ for $i \geq 0,1 \leq j \leq t$, and $X_{i}^{\prime}=\left(K, X_{i}, 1\right)$ for $i \geq 0$. The translation $\tau^{\prime}$ of $\Gamma^{\prime}$ is defined as follows: $\tau^{\prime} Z_{i j}=$ $Z_{i-1, j-1}$ if $i \geq 1, j \geq 2, \tau^{\prime} Z_{i 1}=X_{i-1}$ if $i \geq 1, \tau^{\prime} Z_{0 j}=Y_{j-1}$ if $j \geq 2, Z_{01}$ is projective, $\tau^{\prime} X_{0}^{\prime}=Y_{t}, \tau^{\prime} X_{i}^{\prime}=Z_{i-1, t}$ if $i \geq 1, \tau^{\prime}\left(\tau^{-1} X_{i}\right)=X_{i}^{\prime}$ provided $X_{i}$ is not an injective $A$ module, otherwise $X_{i}^{\prime}$ is injective in $\Gamma^{\prime}$. For the remaining vertices of $\Gamma^{\prime}, \tau^{\prime}$ coincides with the translation of $\Gamma$, or $\Gamma_{D}$, respectively.

If $t=0$ we define the modified algebra $A^{\prime}$ to be the one-point extension $A^{\prime}=A[X]$ and the modified translation quiver $\Gamma^{\prime}$ to be the translation quiver obtained from $\Gamma$ by inserting only the sectional path consisting of the vertices $X_{i}^{\prime}, i \geq 0$.

The non-negative integer $t$ is such that the number of infinite sectional paths parallel to $X_{0} \rightarrow X_{1} \rightarrow X_{2} \rightarrow \cdots$ in the inserted rectangle equals $t+1$. We call $t$ the parameter of the operation.

Since $\Gamma$ is a generalized standard family of components of $\Gamma_{A}$, we then have

Lemma 4.2. $\Gamma^{\prime}$ is a generalized standard family of components of $\Gamma_{A^{\prime}}$.

In case $\Gamma$ is a stable tube, it is clear that any module on the mouth of $\Gamma$ satisfies the condition for being a pivot for the above operation. Actually, the above operation is, in this case, the tube insertion as considered in [28].

(ad 2) Suppose that $\mathscr{S}(X)$ admits two sectional paths starting at $X$, one infinite and the other finite with at least one arrow:

$$
Y_{t} \leftarrow \cdots \leftarrow Y_{2} \leftarrow Y_{1} \leftarrow X=X_{0} \rightarrow X_{1} \rightarrow X_{2} \rightarrow \cdots
$$

where $t \geq 1$. In particular, $X$ is necessarily injective. We define the modified algebra $A^{\prime}$ of $A$ to be the one-point extension $A^{\prime}=A[X]$ and the modified translation quiver $\Gamma^{\prime}$ of $\Gamma$ to be obtained by inserting in $\Gamma$ the rectangle consisting of the modules $Z_{i j}=\left(K, X_{i} \oplus Y_{j},\left[\begin{array}{l}1 \\ 1\end{array}\right]\right)$ for $i \geq 1,1 \leq j \leq t$, and $X_{i}^{\prime}=\left(K, X_{i}, 1\right)$ for $i \geq 1$. The 
translation $\tau^{\prime}$ of $\Gamma^{\prime}$ is defined as follows: $X_{0}^{\prime}$ is projective-injective, $\tau^{\prime} Z_{i j}=Z_{i-1, j-1}$ if $i \geq 2, j \geq 2, \tau^{\prime} Z_{i 1}=X_{i-1}$ if $i \geq 1, \tau^{\prime} Z_{1 j}=Y_{j-1}$ if $j \geq 2, \tau^{\prime} X_{i}^{\prime}=Z_{i-1, t}$ if $i \geq 2, \tau^{\prime} X_{1}^{\prime}=$ $Y_{t}, \tau^{\prime}\left(\tau^{-1} X_{i}\right)=X_{i}^{\prime}$ provided $X_{i}$ is not an injective $A$-module, otherwise $X_{i}^{\prime}$ is injective in $\Gamma^{\prime}$. For the remaining vertices of $\Gamma^{\prime}, \tau^{\prime}$ coincides with the translation $\tau$ of $\Gamma$.

The integer $t \geq 1$ is such that the number of infinite sectional paths parallel to $X_{0} \rightarrow X_{1} \rightarrow X_{2} \rightarrow \cdots$ in the inserted rectangle equals $t+1$. We call $t$ the parameter of the operation.

Since $\Gamma$ is a generalized standard family of components of $\Gamma_{A}$, we then have

Lemma 4.3. $\Gamma^{\prime}$ is a generalized standard family of components of $\Gamma_{A^{\prime}}$.

(ad 3) Assume $\mathscr{S}(X)$ is the mesh-category of two parallel sectional paths:

$$
\begin{gathered}
Y_{1} \rightarrow Y_{2} \rightarrow \cdots \rightarrow Y_{t} \\
\uparrow \\
\uparrow \\
X=X_{0} \rightarrow X_{1} \rightarrow \cdots \rightarrow X_{t-1} \rightarrow X_{t} \rightarrow \cdots
\end{gathered}
$$

where $t \geq 2$. In particular, $X_{t-1}$ is necessarily injective. Moreover, we consider the translation quiver $\bar{\Gamma}$ of $\Gamma$ obtained by deleting the arrows $Y_{i} \rightarrow \tau_{A}^{-1} Y_{i-1}$. We assume that the union $\widehat{\Gamma}$ of connected components of $\bar{\Gamma}$ containing the vertices $\tau_{A}^{-1} Y_{i-1}$, $2 \leq i \leq t$, is a finite translation quiver. Then $\bar{\Gamma}$ is a disjoint union of $\widehat{\Gamma}$ and a cofinite full translation subquiver $\Gamma^{*}$, containing the pivot $X$. We define the modified algebra $A^{\prime}$ of $A$ to be the one-point extension $A^{\prime}=A[X]$ and the modified translation quiver $\Gamma^{\prime}$ of $\Gamma$ to be obtained from $\Gamma^{*}$ by inserting the rectangle consisting of the modules $Z_{i j}=\left(K, X_{i} \oplus Y_{j},\left[\begin{array}{l}1 \\ 1\end{array}\right]\right)$ for $i \geq 1,1 \leq j \leq t$, and $X_{i}^{\prime}=\left(K, X_{i}, 1\right)$ for $i \geq 1$. The translation $\tau^{\prime}$ of $\Gamma^{\prime}$ is defined as follows: $X_{0}^{\prime}$ is projective, $\tau^{\prime} Z_{i j}=Z_{i-1, j-1}$ if $i \geq 2$, $2 \leq j \leq t, \tau^{\prime} Z_{i 1}=X_{i-1}$ if $i \geq 1, \tau^{\prime} X_{i}^{\prime}=Y_{i}$ if $1 \leq i \leq t, \tau^{\prime} X_{i}^{\prime}=Z_{i-1, t}$ if $i \geq t+1$, $\tau^{\prime} Y_{j}=X_{j-2}^{\prime}$ if $2 \leq j \leq t, \tau^{\prime}\left(\tau^{-1} X_{i}\right)=X_{i}^{\prime}$, if $i \geq t$ provided $X_{i}$ is not injective in $\Gamma$, otherwise $X_{i}^{\prime}$ is injective in $\Gamma^{\prime}$. For the remaining vertices of $\Gamma^{\prime}, \tau^{\prime}$ coincides with the translation $\tau$ of $\Gamma^{*}$. We note that $X_{t-1}^{\prime}$ is injective.

The integer $t \geq 2$ is such that the number of infinite sectional paths parallel to $X_{0} \rightarrow X_{1} \rightarrow X_{2} \rightarrow \cdots$ in the inserted rectangle equals $t+1$. We call $t$ the parameter of the operation.

Since $\Gamma$ is a generalized standard family of components of $\Gamma_{A}$, we then have

Lemma 4.4. $\Gamma^{\prime}$ is a generalized standard family of components of $\Gamma_{A^{\prime}}$.

(ad 4) Suppose that $\mathscr{S}(X)$ consists an infinite sectional path, starting at $X$

$$
X=X_{0} \rightarrow X_{1} \rightarrow X_{2} \rightarrow \cdots
$$

and

$$
Y=Y_{1} \rightarrow Y_{2} \rightarrow \cdots \rightarrow Y_{t}
$$

with $t \geq 1$, be a finite sectional path in $\Gamma_{A}$. Let $r$ be a positive integer. Moreover, we consider the translation quiver $\bar{\Gamma}$ of $\Gamma$ obtained by deleting the arrows $Y_{i} \rightarrow \tau_{A}^{-1} Y_{i-1}$. 
We assume that the union $\widehat{\Gamma}$ of connected components of $\bar{\Gamma}$ containing the vertices $\tau_{A}^{-1} Y_{i-1}, 2 \leq i \leq t$, is a finite translation quiver. Then $\bar{\Gamma}$ is a disjoint union of $\widehat{\Gamma}$ and a cofinite full translation subquiver $\Gamma^{*}$, containing the pivot $X$. For $r=0$ we define the modified algebra $A^{\prime}$ of $A$ to be the one-point extension $A^{\prime}=A[X \oplus Y]$ and the modified translation quiver $\Gamma^{\prime}$ of $\Gamma$ to be obtained from $\Gamma^{*}$ by inserting the rectangle consisting of the modules $Z_{i j}=\left(K, X_{i} \oplus Y_{j},\left[\begin{array}{l}1 \\ 1\end{array}\right]\right)$ for $i \geq 0,1 \leq j \leq t$, and $X_{i}^{\prime}=\left(K, X_{i}, 1\right)$ for $i \geq 1$. The translation $\tau^{\prime}$ of $\Gamma^{\prime}$ is defined as follows: $\tau^{\prime} Z_{i j}=$ $Z_{i-1, j-1}$ if $i \geq 1, j \geq 2, \tau^{\prime} Z_{i 1}=X_{i-1}$ if $i \geq 1, \tau^{\prime} Z_{0 j}=Y_{j-1}$ if $j \geq 2, Z_{01}$ is projective, $\tau^{\prime} X_{0}^{\prime}=Y_{t}, \tau^{\prime} X_{i}^{\prime}=Z_{i-1, t}$ if $i \geq 1, \tau^{\prime}\left(\tau^{-1} X_{i}\right)=X_{i}^{\prime}$ provided $X_{i}$ is not injective in $\Gamma$, otherwise $X_{i}^{\prime}$ is injective in $\Gamma^{\prime}$. For the remaining vertices of $\Gamma^{\prime}, \tau^{\prime}$ coincides with the translation of $\Gamma^{*}$.

For $r \geq 1$, let $G=T_{r}(K), U_{1, t+1}, U_{2, t+1}, \ldots, U_{r, t+1}$ denote the indecomposable projective $G$-modules, $U_{r, t+1}, U_{r, t+2}, \ldots, U_{r, t+r}$ denote the indecomposable injective $G$-modules, with $U_{r, t+1}$ the unique indecomposable projective-injective $G$-module. We define the modified algebra $A^{\prime}$ of $A$ to be the triangular matrix algebra of the form:

$$
A^{\prime}=\left[\begin{array}{cccccc}
A & 0 & 0 & \ldots & 0 & 0 \\
Y & K & 0 & \ldots & 0 & 0 \\
Y & K & K & \ldots & 0 & 0 \\
\vdots & \vdots & \vdots & \ddots & \vdots & \vdots \\
Y & K & K & \ldots & K & 0 \\
X \oplus Y & K & K & \ldots & K & K
\end{array}\right]
$$

with $r+2$ columns and rows and the modified translation quiver $\Gamma^{\prime}$ of $\Gamma$ to be obtained from $\Gamma^{*}$ by inserting the rectangles consisting of the modules $U_{k l}=Y_{l} \oplus$ $U_{k, t+k}$ for $1 \leq k \leq r, 1 \leq l \leq t$, and $Z_{i j}=\left(K, X_{i} \oplus U_{r j},\left[\begin{array}{l}1 \\ 1\end{array}\right]\right)$ for $i \geq 0,1 \leq j \leq$ $t+r$, and $X_{i}^{\prime}=\left(K, X_{i}, 1\right)$ for $i \geq 0$. The translation $\tau^{\prime}$ of $\Gamma^{\prime}$ is defined as follows: $\tau^{\prime} Z_{i j}=Z_{i-1, j-1}$ if $i \geq 1, j \geq 2, \tau^{\prime} Z_{i 1}=X_{i-1}$ if $i \geq 1, \tau^{\prime} Z_{0 j}=U_{r, j-1}$ if $2 \leq j \leq t+r$, $Z_{01}, U_{k 1}, 1 \leq k \leq r$ are projective, $\tau^{\prime} U_{k l}=U_{k-1, l-1}$ if $2 \leq k \leq r, 2 \leq l \leq t+r, \tau^{\prime} U_{1 l}=$ $Y_{l-1}$ if $2 \leq l \leq t+1, \tau^{\prime} X_{0}^{\prime}=U_{r, t+r}, \tau^{\prime} X_{i}^{\prime}=Z_{i-1, t+r}$ if $i \geq 1, \tau^{\prime}\left(\tau^{-1} X_{i}\right)=X_{i}^{\prime}$ provided $X_{i}$ is not injective in $\Gamma$, otherwise $X_{i}^{\prime}$ is injective in $\Gamma^{\prime}$. For the remaining vertices of $\Gamma^{\prime}, \tau^{\prime}$ coincides with the translation of $\Gamma^{*}$, or $\Gamma_{G}$, respectively.

We note that the quiver $Q_{A^{\prime}}$ of $A^{\prime}$ is obtained from the quiver of the double onepoint extension $A[X][Y]$ by adding a path of length $r+1$ with source at the extension vertex of $A[X]$ and sink at the extension vertex of $A[Y]$.

The integers $t \geq 1$ and $r \geq 0$ are such that the number of infinite sectional paths parallel to $X_{0} \rightarrow X_{1} \rightarrow X_{2} \rightarrow \cdots$ in the inserted rectangles equals $t+r+1$. We call $t+r$ the parameter of the operation.

Since $\Gamma$ is a generalized standard family of components of $\Gamma_{A}$, we then have

Lemma 4.5. $\Gamma^{\prime}$ is a generalized standard family of components of $\Gamma_{A^{\prime}}$.

(fad 1) Assume $\mathscr{S}(X)$ consists of a finite sectional path starting at $X$ :

$$
X=X_{0} \rightarrow X_{1} \rightarrow X_{2} \rightarrow \cdots \rightarrow X_{s}
$$


where $s \geq 0$ and $X_{s}$ is injective. Let $t \geq 1$ be a positive integer, $D=T_{t}(K)$ and $Y_{1}$, $Y_{2}, \ldots, Y_{t}$ denote the indecomposable injective $D$-modules with $Y=Y_{1}$ the unique indecomposable projective-injective $D$-module. We define the modified algebra $A^{\prime}$ of $A$ to be the one-point extension

$$
A^{\prime}=(A \times D)[X \oplus Y]
$$

and the modified translation quiver $\Gamma^{\prime}$ of $\Gamma$ to be obtained by inserting in $\Gamma$ the rectangle consisting of the modules $Z_{i j}=\left(K, X_{i} \oplus Y_{j},\left[\begin{array}{l}1 \\ 1\end{array}\right]\right)$ for $0 \leq i \leq s, 1 \leq j \leq t$, $X_{i}^{\prime}=\left(K, X_{i}, 1\right)$ for $0 \leq i \leq s, Y_{j}^{\prime}=\left(K, Y_{j}, 1\right)$ for $1 \leq j \leq t$, and $W=S_{p}$, where $p$ is the extension vertex of $A[X]$. The translation $\tau^{\prime}$ of $\Gamma^{\prime}$ is defined as follows: $\tau^{\prime} Z_{i j}=Z_{i-1, j-1}$ if $i \geq 1, j \geq 2, \tau^{\prime} Z_{i 1}=X_{i-1}$ if $i \geq 1, \tau^{\prime} Z_{0 j}=Y_{j-1}$ if $j \geq 2, Z_{01}$ is projective, $\tau^{\prime} X_{0}^{\prime}=Y_{t}, \tau^{\prime} X_{i}^{\prime}=Z_{i-1, t}$ if $i \geq 1, \tau^{\prime}\left(\tau^{-1} X_{i}\right)=X_{i}^{\prime}$ provided $X_{i}$ is not injective in $\Gamma$, otherwise $X_{i}^{\prime}$ is injective in $\Gamma^{\prime}, \tau^{\prime} Y_{1}^{\prime}=X_{s}, \tau^{\prime} Y_{j}^{\prime}=Z_{s, j-1}$ if $2 \leq j \leq t, \tau^{\prime} W=Z_{s t}$. For the remaining vertices of $\Gamma^{\prime}, \tau^{\prime}$ coincides with the translation of $\Gamma$, or $\Gamma_{D}$, respectively. If $t=0$ we define the modified algebra $A^{\prime}$ to be the one-point extension $A^{\prime}=A[X]$ and the modified translation quiver $\Gamma^{\prime}$ to be the component obtained from $\Gamma$ by inserting only the sectional path consisting of the vertices $X_{i}^{\prime}, 0 \leq i \leq s$, and $W$.

Observe that for $s=0=t$ the new translation quiver $\Gamma^{\prime}$ is obtained from $\Gamma$ by adding the projective-injective vertex $X_{0}^{\prime}$ and the vertex $W$ with $\tau^{\prime} W=X_{0}$.

(fad 2) Suppose that $\mathscr{S}(X)$ admits two finite sectional paths starting at $X$, each of them with at least one arrow:

$$
Y_{t} \leftarrow \cdots \leftarrow Y_{2} \leftarrow Y_{1} \leftarrow X=X_{0} \rightarrow X_{1} \rightarrow X_{2} \rightarrow \cdots \rightarrow X_{s}
$$

where $t \geq 1$ and $s \geq 1$. In particular, $X$ and $X_{s}$ are necessarily injective. We define the modified algebra $A^{\prime}$ of $A$ to be the one-point extension $A^{\prime}=A[X]$ and the modified translation quiver $\Gamma^{\prime}$ of $\Gamma$ to be obtained by inserting in $\Gamma$ the rectangle consisting of the modules $Z_{i j}=\left(K, X_{i} \oplus Y_{j},\left[\begin{array}{l}1 \\ 1\end{array}\right]\right)$ for $1 \leq i \leq s, 1 \leq j \leq t, X_{i}^{\prime}=\left(K, X_{i}, 1\right)$ for $1 \leq i \leq s, Y_{j}^{\prime}=\left(K, Y_{j}, 1\right)$ for $1 \leq j \leq t$, and $W=S_{p}$, where $p$ is the extension vertex of $A[X]$. The translation $\tau^{\prime}$ of $\Gamma^{\prime}$ is defined as follows: $X_{0}^{\prime}$ is projective-injective, $\tau^{\prime} Z_{i j}=$ $Z_{i-1, j-1}$ if $i \geq 2, j \geq 2, \tau^{\prime} Z_{i 1}=X_{i-1}$ if $i \geq 1, \tau^{\prime} Z_{1 j}=Y_{j-1}$ if $j \geq 2, \tau^{\prime} X_{i}^{\prime}=Z_{i-1, t}$ if $i \geq 2, \tau^{\prime} X_{1}^{\prime}=Y_{t}, \tau^{\prime}\left(\tau^{-1} X_{i}\right)=X_{i}^{\prime}$ provided $X_{i}$ is not injective in $\Gamma$, otherwise $X_{i}^{\prime}$ is injective in $\Gamma^{\prime}, \tau^{\prime} Y_{1}^{\prime}=X_{s}, \tau^{\prime} Y_{j}^{\prime}=Z_{s, j-1}$ if $2 \leq j \leq t, \tau^{\prime} W=Z_{s t}$. For the remaining vertices of $\Gamma^{\prime}, \tau^{\prime}$ coincides with the translation $\tau$ of $\Gamma$.

(fad 3) Assume $\mathscr{S}(X)$ is the mesh-category of two finite parallel sectional paths:

$$
\begin{gathered}
Y_{1} \rightarrow Y_{2} \rightarrow \cdots \rightarrow Y_{t} \\
\uparrow \\
\uparrow \\
X=X_{0} \rightarrow X_{1} \rightarrow \cdots \rightarrow X_{t-1} \rightarrow X_{t} \rightarrow \cdots \rightarrow X_{S}
\end{gathered}
$$

where $s \geq t-1, t \geq 2$. In particular, $X_{t-1}$ and $X_{s}$ are necessarily injective. We define the modified algebra $A^{\prime}$ of $A$ to be the one-point extension $A^{\prime}=A[X]$ and the modified 
translation quiver $\Gamma^{\prime}$ of $\Gamma$ to be obtained by inserting in $\Gamma$ the rectangle consisting of the modules $Z_{i j}=\left(K, X_{i} \oplus Y_{j},\left[\begin{array}{l}1 \\ 1\end{array}\right]\right)$ for $1 \leq i \leq s, 1 \leq j \leq t, X_{i}^{\prime}=\left(K, X_{i}, 1\right)$ for $1 \leq i \leq s, Y_{j}^{\prime}=\left(K, Y_{j}, 1\right)$ for $1 \leq j \leq t$, and $W=S_{p}$, where $p$ is the extension vertex of $A[X]$. The translation $\tau^{\prime}$ of $\Gamma^{\prime}$ is defined as follows: $X_{0}^{\prime}$ is projective, $\tau^{\prime} Z_{i j}=Z_{i-1, j-1}$ if $i \geq 2,2 \leq j \leq t, \tau^{\prime} Z_{i 1}=X_{i-1}$ if $i \geq 1, \tau^{\prime} X_{i}^{\prime}=Y_{i}$ if $1 \leq i \leq t, \tau^{\prime} X_{i}^{\prime}=Z_{i-1, t}$ if $i \geq$ $t+1, \tau^{\prime} Y_{j}=X_{j-2}^{\prime}$ if $2 \leq j \leq t, \tau^{\prime}\left(\tau^{-1} X_{i}\right)=X_{i}^{\prime}$, if $i \geq t$ provided $X_{i}$ is not injective in $\Gamma$, otherwise $X_{i}^{\prime}$ is injective in $\Gamma^{\prime}$. In both cases, $X_{t-1}^{\prime}$ is injective, $\tau^{\prime} Y_{1}^{\prime}=X_{s}$, $\tau^{\prime} Y_{j}^{\prime}=Z_{s, j-1}$ if $2 \leq j \leq t, \tau^{\prime} W=Z_{s t}$. For the remaining vertices of $\Gamma^{\prime}, \tau^{\prime}$ coincides with the translation $\tau$ of $\Gamma$. Observe that for $s=t-1$ we have $Z_{t t}=Y_{t}^{\prime}$ and $X_{t}^{\prime}=W$.

(fad 4) Suppose that $\mathscr{S}(X)$ consists of a finite sectional path starting at $X$ :

$$
X=X_{0} \rightarrow X_{1} \rightarrow X_{2} \rightarrow \cdots \rightarrow X_{s}
$$

with $s \geq 1$ and $X_{s}$ injective, and

$$
Y=Y_{1} \rightarrow Y_{2} \rightarrow \cdots \rightarrow Y_{t}
$$

$t \geq 1$, be a finite sectional path in $\Gamma_{A}$. Let $r$ be a positive integer. For $r=0$ we define the modified algebra $A^{\prime}$ of $A$ to be the one-point extension $A^{\prime}=A[X \oplus Y]$ and the modified translation quiver $\Gamma^{\prime}$ of $\Gamma$ to be obtained by inserting in $\Gamma$ the rectangle consisting of the modules $Z_{i j}=\left(K, X_{i} \oplus Y_{j},\left[\begin{array}{l}1 \\ 1\end{array}\right]\right)$ for $0 \leq i \leq s, 1 \leq j \leq t$, $X_{i}^{\prime}=\left(K, X_{i}, 1\right)$ for $0 \leq i \leq s, Y_{j}^{\prime}=\left(K, Y_{j}, 1\right)$ for $1 \leq j \leq t$, and $W=S_{p}$, where $p$ is the extension vertex of $A[X]$. The translation $\tau^{\prime}$ of $\Gamma^{\prime}$ is defined as follows: $\tau^{\prime} Z_{i j}=$ $Z_{i-1, j-1}$ if $i \geq 1, j \geq 2, \tau^{\prime} Z_{i 1}=X_{i-1}$ if $i \geq 1, \tau^{\prime} Z_{0 j}=Y_{j-1}$ if $j \geq 2, Z_{01}$ is projective, $\tau^{\prime} X_{0}^{\prime}=Y_{t}, \tau^{\prime} X_{i}^{\prime}=Z_{i-1, t}$ if $i \geq 1, \tau^{\prime}\left(\tau^{-1} X_{i}\right)=X_{i}^{\prime}$ provided $X_{i}$ is not injective in $\Gamma$, otherwise $X_{i}^{\prime}$ is injective in $\Gamma^{\prime}, \tau^{\prime} Y_{1}^{\prime}=X_{s}, \tau^{\prime} Y_{j}^{\prime}=Z_{s, j-1}$ if $2 \leq j \leq t, \tau^{\prime} W=Z_{s t}$. For the remaining vertices of $\Gamma^{\prime}, \tau^{\prime}$ coincides with the translation of $\Gamma$.

For $r \geq 1$, let $G=T_{r}(K), U_{1, t+1}, U_{2, t+1}, \ldots, U_{r, t+1}$ denote the indecomposable projective $G$-modules, $U_{r, t+1}, U_{r, t+2}, \ldots, U_{r, t+r}$ denote the indecomposable injective $G$-modules, with $U_{r, t+1}$ the unique indecomposable projective-injective $G$-module. We define the modified algebra $A^{\prime}$ of $A$ to be the triangular matrix algebra of the form:

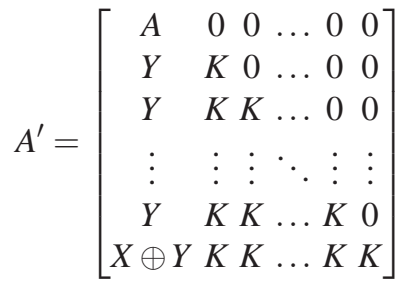

with $r+2$ columns and rows and the modified translation quiver $\Gamma^{\prime}$ of $\Gamma$ to be obtained by inserting in $\Gamma$ the rectangles consisting of the modules $U_{k l}=Y_{l} \oplus U_{k, t+k}$ for $1 \leq k \leq r, 1 \leq l \leq t, Z_{i j}=\left(K, X_{i} \oplus U_{r j},\left[\begin{array}{l}1 \\ 1\end{array}\right]\right)$ for $0 \leq i \leq s, 1 \leq j \leq t+r$, $X_{i}^{\prime}=\left(K, X_{i}, 1\right)$ for $0 \leq i \leq s, Y_{j}^{\prime}=\left(K, U_{r j}, 1\right)$ for $1 \leq j \leq t+r$, and $W=S_{p}$, where 
$p$ is the extension vertex of $A[X]$. The translation $\tau^{\prime}$ of $\Gamma^{\prime}$ is defined as follows: $\tau^{\prime} Z_{i j}=Z_{i-1, j-1}$ if $i \geq 1, j \geq 2, \tau^{\prime} Z_{i 1}=X_{i-1}$ if $i \geq 1, \tau^{\prime} Z_{0 j}=U_{r, j-1}$ if $2 \leq j \leq t+r$, $Z_{01}, U_{k 1}, 1 \leq k \leq r$ are projective, $\tau^{\prime} U_{k l}=U_{k-1, l-1}$ if $2 \leq k \leq r, 2 \leq l \leq t+r, \tau^{\prime} U_{1 l}=$ $Y_{l-1}$ if $2 \leq l \leq t+1, \tau^{\prime} X_{0}^{\prime}=U_{r, t+r}, \tau^{\prime} X_{i}^{\prime}=Z_{i-1, t+r}$ if $i \geq 1, \tau^{\prime}\left(\tau^{-1} X_{i}\right)=X_{i}^{\prime}$ provided $X_{i}$ is not injective in $\Gamma$, otherwise $X_{i}^{\prime}$ is injective in $\Gamma^{\prime}, \tau^{\prime} Y_{1}^{\prime}=X_{s}, \tau^{\prime} Y_{j}^{\prime}=Z_{s, j-1}$ if $2 \leq j \leq t+r, \tau^{\prime} W=Z_{s, t+r}$. For the remaining vertices of $\Gamma^{\prime}, \tau^{\prime}$ coincides with the translation of $\Gamma$, or $\Gamma_{G}$, respectively.

(ad 5) We define the modified algebra $A^{\prime}$ of $A$ to be the iteration of the extensions described in the definitions of the admissible operations $(\operatorname{ad} 1),(\operatorname{ad} 2),(\operatorname{ad} 3),(\operatorname{ad} 4)$, and their finite versions corresponding to the operations (fad 1), (fad 2), (fad 3$)$ and (fad 4). The modified translation quiver $\Gamma^{\prime}$ of $\Gamma$ is obtained in the following three steps: first we are doing on $\Gamma$ one of the operations (fad 1), (fad 2) or (fad 3), next a finite number (possibly empty) of the operation (fad 4) and finally the operation (ad 4), and in such a way that the sectional paths starting from all the new projective vertices have a common cofinite (infinite) sectional subpath.

Since $\Gamma$ is a generalized standard family of components of $\Gamma_{A}$, we then have

Lemma 4.6. $\Gamma^{\prime}$ is a generalized standard family of components of $\Gamma_{A^{\prime}}$.

Finally, together with each of the admissible operations $(\operatorname{ad} 1),(\operatorname{ad} 2),(\operatorname{ad} 3)$, $(\operatorname{ad} 4)$ and $(\operatorname{ad} 5)$, we consider its dual, denoted by $\left(\operatorname{ad} 1^{*}\right),\left(\operatorname{ad} 2^{*}\right),\left(\operatorname{ad} 3^{*}\right),\left(\operatorname{ad} 4^{*}\right)$ and $\left(\operatorname{ad} 5^{*}\right)$. These ten operations are called the admissible operations. Following [50] a connected translation quiver $\Gamma$ is said to be a generalized multicoil if $\Gamma$ can be obtained from a finite family $\mathscr{T}_{1}, \mathscr{T}_{2}, \ldots, \mathscr{T}_{S}$ of stable tubes by an iterated application of admissible operations $(\operatorname{ad} 1),\left(\operatorname{ad} 1^{*}\right),(\operatorname{ad} 2),\left(\operatorname{ad} 2^{*}\right),(\operatorname{ad} 3),\left(\operatorname{ad} 3^{*}\right),(\operatorname{ad} 4),\left(\operatorname{ad} 4^{*}\right)$, (ad 5) or $\left(\operatorname{ad~} 5^{*}\right)$. If $s=1$, such a translation quiver $\Gamma$ is said to be a generalized coil. The admissible operations of types $(\operatorname{ad} 1),(\operatorname{ad} 2),(\operatorname{ad} 3),\left(\operatorname{ad} 1^{*}\right),\left(\operatorname{ad} 2^{*}\right)$ and $\left(\operatorname{ad} 3^{*}\right)$ have been introduced in [5], [7], [8], and the admissible operations $(\operatorname{ad} 4)$ and $\left(\operatorname{ad~} 4^{*}\right)$ for $r=0$ in [45].

Observe that any stable tube is trivially a generalized coil. A tube (in the sense of [28]) is a generalized coil having the property that each admissible operation in the sequence defining it is of the form ( $\mathrm{ad} 1)$ or $\left(\mathrm{ad} 1^{*}\right)$. If we apply only operations of type (ad 1) (respectively, of type $\left.\left(\operatorname{ad~} 1^{*}\right)\right)$ then such a generalized coil is called a ray tube (respectively, a coray tube). Observe that a generalized coil without injective (respectively, projective) vertices is a ray tube (respectively, a coray tube). A quasitube (in the sense of [70]) is a generalized coil having the property that each of the admissible operations in the sequence defining it is of type $(\operatorname{ad} 1),\left(\operatorname{ad~} 1^{*}\right),(\operatorname{ad} 2)$ or (ad 2*). Finally, following [7] a coil is a generalized coil having the property that each of the admissible operations in the sequence defining it is one of the forms $(\operatorname{ad} 1),\left(\operatorname{ad} 1^{*}\right),(\operatorname{ad} 2),\left(\operatorname{ad} 2^{*}\right),(\operatorname{ad} 3)$ or $\left(\operatorname{ad} 3^{*}\right)$. We note that any generalized multicoil $\Gamma$ is a coherent translation quiver with trivial valuations and its cyclic part ${ }_{c} \Gamma$ (the translation subquiver of $\Gamma$ obtained by removing from $\Gamma$ all acyclic vertices and the arrows attached to them) is infinite, connected and cofinite in $\Gamma$, and so $\Gamma$ is almost cyclic.

Let $C$ be the product $C_{1} \times \ldots \times C_{m}$ of a family $C_{1}, \ldots, C_{m}$ of tame concealed algebras and $\mathscr{T}^{C}$ the disjoin union $\mathscr{T}^{C_{1}} \cup \ldots \cup \mathscr{T}^{C_{m}}$ of $\mathbb{P}_{1}(K)$-families $\mathscr{T}^{C_{1}}, \ldots, \mathscr{T}^{C_{m}}$ of 
pairwise orthogonal generalized standard stable tubes of $\Gamma_{C_{1}}, \ldots, \Gamma_{C_{m}}$, respectively. Following [51], we say that an algebra $A$ is a generalized multicoil enlargement of $C_{1}, \ldots, C_{m}$ if $A$ is obtained from $C$ by an iteration of admissible operations of types $(\operatorname{ad} 1)-(\operatorname{ad} 5)$ and $\left(\operatorname{ad~} 1^{*}\right)-\left(\operatorname{ad~} 5^{*}\right)$ performed either on stable tubes of $\mathscr{T}^{C}$ or on generalized multicoils obtained from stable tubes of $\mathscr{T}^{C}$ by means of operations done so far. It follows from [51, Corollary B] that then $A$ is a triangular algebra, and hence the Tits and Euler forms $q_{A}$ and $\chi_{A}$ of $A$ are defined. In fact, in [51] generalized multicoil enlargements of finite families of arbitrary concealed canonical algebras have been introduced and investigated. But in the tame case we may restrict to the generalized multicoil enlargements of tame concealed algebras. Namely, we have the following consequence of [51, Theorems A and F].

Theorem 4.7. Let $A$ be an algebra. The following statements are equivalent:

(i) $A$ is tame and $\Gamma_{A}$ admits a separating family of almost cyclic coherent components.

(ii) A is a tame generalized multicoil enlargement of a finite family of tame concealed algebras.

(iii) $A$ is a generalized multicoil enlargement of a finite family of tame concealed algebras and the Tits form $q_{A}$ is weakly nonnegative.

From now on, by a tame generalized multicoil algebra we mean a connected tame generalized multicoil enlargement of a finite family of tame concealed algebras. The following consequence of [51, Theorems $\mathrm{C}$ and F] describes the structure of the Auslander-Reiten quivers of tame generalized multicoil algebras.

Theorem 4.8. Let A be a tame generalized multicoil algebra obtained from a family $C_{1}, \ldots, C_{m}$ of tame concealed algebras. There are convex subcategories $A^{(l)}=A_{1}^{(l)} \times$ $\ldots \times A_{m}^{(l)}$ and $A^{(r)}=A_{1}^{(r)} \times \ldots \times A_{m}^{(r)}$ of $A$ such that the following statement hold:

(i) For each $i \in\{1, \ldots, m\}, A_{i}^{(l)}$ is a domestic tubular or tubular coextension of the tame concealed algebra $C_{i}$.

(ii) For each $i \in\{1, \ldots, m\}, A_{i}^{(r)}$ is a domestic tubular or tubular extension of the tame concealed algebra $C_{i}$.

(iii) The Auslander-Reiten quiver $\Gamma_{A}$ of $A$ is of the form

$$
\Gamma_{A}=\mathscr{P}^{A} \cup \mathscr{C}^{A} \cup \mathscr{Q}^{A},
$$

where $\mathscr{C}^{A}$ is a family of generalized multicoils separating $\mathscr{P}^{A}$ from $\mathscr{Q}^{A}$ such that:

(1) $\mathscr{C}^{A}$ is obtained from the $\mathbb{P}_{1}(K)$-families $\mathscr{T}^{C_{1}}, \ldots, \mathscr{T}^{C_{m}}$ of stable tubes of $\Gamma_{C_{1}}, \ldots, \Gamma_{C_{m}}$ by admissible operations corresponding to the admissible operations leading from $C_{1}, \ldots, C_{m}$ to $A$;

(2) $\mathscr{P}^{A}$ is the disjoint union $\mathscr{P}_{1}^{A_{1}^{(l)}} \cup \ldots \cup \mathscr{P}^{A_{m}^{(l)}}$, where, for each $i \in\{1, \ldots, m\}, \mathscr{P}_{i}^{(l)}$ is either the postprojective component of $\Gamma_{A_{i}^{(l)}}$, if $A_{i}^{(l)}$ is tilted of Euclidean type, or $\mathscr{P} A_{i}^{(l)}=\mathscr{P}_{0}^{A_{i}^{(l)}} \cup \mathscr{T}_{0}^{A_{i}^{(l)}} \cup\left(\bigcup_{q \in \mathbb{Q}^{+}} \mathscr{T}_{q}^{A_{i}^{(l)}}\right)$, if $A_{i}^{(l)}$ is a tubular algebra; 
(3) $\mathscr{Q}^{A}$ is the disjoint union $\mathscr{Q}_{1}^{A_{1}^{(r)}} \cup \ldots \cup \mathscr{Q}^{A_{m}^{(r)}}$, where, for each $i \in\{1, \ldots, m\}, \mathscr{Q}^{A_{i}^{(r)}}$ is either the preinjective component of $\Gamma_{A_{i}^{(r)}}$, if $A_{i}^{(r)}$ is tilted of Euclidean type, or $\mathscr{Q}^{A_{i}^{(r)}}=\left(\bigcup_{q \in \mathbb{Q}^{+}} \mathscr{T}_{q}^{A_{i}^{(r)}}\right) \cup \mathscr{T}_{\infty}^{A_{i}^{(r)}} \cup \mathscr{Q}_{\infty}^{A_{i}^{(r)}}$, if $A_{i}^{(r)}$ is a tubular algebra.

In particular, we have the following consequence of Theorems 3.1 and 4.8

Corollary 4.9. Let A be a tame generalized multicoil algebra. Then A is cycle-finite.

Further, as a consequence of Theorems 3.4 and 4.8 , we obtain the following fact.

Corollary 4.10. Let $A$ be a tame generalized multicoil algebra and $\Gamma_{A}=\mathscr{P} A \cup \mathscr{C}^{A} \cup$ $\mathscr{Q}^{A}$ the canonical decomposition of $\Gamma_{A}$. The following statements are equivalent:

(i) A is domestic.

(ii) $\mathscr{P}^{A}$ is a disjoint union of postprojective components of Euclidean type and $\mathscr{Q}^{A}$ is a disjoint union of preinjective components of Euclidean type.

Moreover, the following consequence of [51, Theorem E] describes the homological properties of modules over tame generalized multicoil algebras.

Theorem 4.11. Let A be a tame generalized multicoil algebra and $\Gamma_{A}=\mathscr{P}^{A} \cup \mathscr{C}^{A} \cup$ $\mathscr{Q}^{A}$ the canonical decomposition of $\Gamma_{A}$ described above. Then the following statements hold:

(i) $\mathrm{pd}_{A} X \leq 1$ for any module $X$ in $\mathscr{P}^{A}$.

(ii) $\operatorname{id}_{A} X \leq 1$ for any module $X$ in $\mathscr{Q}^{A}$.

(iii) $\operatorname{pd}_{A} X \leq 2$ and $\operatorname{id}_{A} X \leq 2$ for any module $X$ in $\mathscr{C}^{A}$.

(iv) $\operatorname{gl} \operatorname{dim} A \leq 3$.

\section{Tame generalized double tilted algebras}

In this section we introduce and describe basic properties of the class of tame generalized double tilted algebras, which is the class of tame algebras in the class of generalized double tilted algebras investigated in [62], [63], [79].

Let $H$ be a hereditary algebra, $T$ a (multiplicity-free) tilting module in $\bmod H$ and $B=\operatorname{End}_{H}(T)$ the associated tilted algebra. Then $T$ induces the torsion pair $(\mathscr{T}(T), \mathscr{F}(T))$ in $\bmod H$, with the torsion class $\mathscr{T}(T)=\left\{M \in \bmod H ; \operatorname{Ext}_{A}^{1}(T, M)=\right.$ $0\}$ and the torsion-free class $\mathscr{T}(F)=\left\{N \in \bmod H ; \operatorname{Hom}_{H}(T, N)=0\right\}$, and the torsion pair $(\mathscr{X}(T), \mathscr{Y}(T))$ in $\bmod B$, with the torsion class $\mathscr{X}(T)=\left\{X \in \bmod B ; X \otimes_{B}\right.$ $T=0\}$ and the torsion-free class $\mathscr{Y}(T)=\left\{Y \in \bmod H ; \operatorname{Tor}_{1}^{B}(Y, T)=0\right\}$. Then, by the Brenner-Butler theorem, the functors $\operatorname{Hom}_{A}(T,-)$ and $-\otimes_{B} T$ induce quasiinverse equivalence between $\mathscr{T}(T)$ and $\mathscr{Y}(T)$, and the functors $\operatorname{Ext}_{A}^{1}(T,-)$ and $\operatorname{Tor}_{1}^{B}(-, T)$ induce quasi-inverse equivalence between $\mathscr{F}(T)$ and $\mathscr{X}(T)$ (see [23], [33]). Moreover, $(\mathscr{X}(T), \mathscr{Y}(T))$ is a splitting torsion pair of $\bmod B$, that is, every indecomposable module in $\bmod B$ belongs either to $\mathscr{X}(T)$ or $\mathscr{Y}(T)$. Further, the 
images $\operatorname{Hom}_{H}(T, I)$ of the indecomposable injective $H$-modules $I$ via the functor $\operatorname{Hom}_{H}(T, I)$ form a section $\Sigma$ of an acyclic component $\mathscr{C}_{T}$ of $\Gamma_{B}$ such that $\Sigma$ is isomorphic to the opposite quiver $Q_{H}^{\text {op }}$ of the quiver $Q_{H}$ of $H$, any predecessor of $\Sigma$ in $\mathscr{C}_{T}$ lies in $\mathscr{Y}(T)$, and any proper successor of $\Sigma$ in $\mathscr{C}_{T}$ lies in $\mathscr{X}(T)$. Therefore, the component $\mathscr{C}_{T}$ of $\Gamma_{B}$ connects the torsion-free part $\mathscr{Y}(T)$ with the torsion part $\mathscr{X}(T)$ along the section $\Sigma$, and hence $\mathscr{C}_{T}$ is called the connecting component of $\Gamma_{B}$ determined by $T$.

The following theorem proved independently by Liu [43] and Skowroński [72] gives a handy criterion for an algebra to be a tilted algebra.

Theorem 5.1. An algebra $B$ is a tilted algebra if and only if $\Gamma_{B}$ contains a component $\mathscr{C}$ with a faithful section $\Sigma$ such that $\operatorname{Hom}_{B}\left(U, \tau_{B} V\right)=0$ for all modules $U, V$ from $\Sigma$. Moreover, in this case, the direct sum $T$ of all modules on $\Sigma$ is a tilting $B$-module, $H=\operatorname{End}_{B}(T)$ is a hereditary algebra, $T^{*}=D\left({ }_{H} T\right)$ is a tilting $H$-module with $B \cong$ $\operatorname{End}_{H}\left(T^{*}\right)$, and $\mathscr{C}$ is the connecting component $\mathscr{C}_{T^{*}}$ of $\Gamma_{B}$ determined by $T^{*}$.

The general shape of the Auslander-Reiten quiver of a tilted algebra has been described by Kerner in [37]. We will describe only the Auslander-Reiten quivers of tame tilted algebras, which are exactly the cycle-finite tilted algebras.

Theorem 5.2. Let $H=K \Delta$ be a hereditary algebra, $T$ a tilting $H$-module, and assume that the associated tilted algebra $B=\operatorname{End}_{H}(T)$ is tame. Then the connecting component $\mathscr{C}_{T}$ of $\Gamma_{B}$ determined by $T$ admits a finite (possibly empty) family of pairwise disjoint translation subquivers $\mathscr{D}_{1}^{(l)}, \ldots, \mathscr{D}_{m}^{(l)}, \mathscr{D}_{1}^{(r)}, \ldots, \mathscr{D}_{n}^{(r)}$ such that the following statements hold.

(i) For each $i \in\{1, \ldots, m\}$, there exists an isomorphism of translation quivers $\mathscr{D}_{i}^{(l)} \cong$ $\mathbb{N} \Delta_{i}^{(l)}$, where $\Delta_{i}^{(l)}$ is a connected convex subquiver of $\Delta$ of Euclidean type and $\mathscr{D}_{i}^{(l)}$ is closed under predecessors in $\mathscr{C}_{T}$.

(ii) For each $j \in\{1, \ldots, n\}$, there exists an isomorphism of translation quivers $\mathscr{D}_{j}^{(r)} \cong$ $(-\mathbb{N}) \Delta_{j}^{(r)}$, where $\Delta_{j}^{(r)}$ is a connected convex subquiver of $\Delta$ of Euclidean type and $\mathscr{D}_{j}^{(r)}$ is closed under successors in $\mathscr{C}_{T}$.

(iii) All but finitely many modules of $\mathscr{C}_{T}$ lie in $\mathscr{D}_{1}^{(l)} \cup \cdots \cup \mathscr{D}_{m}^{(l)} \cup \mathscr{D}_{1}^{(r)} \cup \cdots \cup \mathscr{D}_{n}^{(r)}$.

(iv) For each $i \in\{1, \ldots, m\}$, there exists a tilted algebra $B_{i}^{(l)}=\operatorname{End}_{H_{i}^{(l)}}\left(T_{i}^{(l)}\right)$, where $H_{i}^{(l)}$ is the path algebra $K \Delta_{i}^{(l)}, T_{i}^{(l)}$ is a tilting $H_{i}^{(l)}$-module without nonzero preinjective direct summands, $B_{i}^{(l)}$ is a quotient algebra of $B$, and $\mathscr{D}_{i}^{(l)}$ coincides with the torsion-free part $\mathscr{Y}\left(T_{i}^{(l)}\right) \cap \mathscr{C}_{T_{i}^{(l)}}$ of the connecting component $\mathscr{C}_{T_{i}^{(l)}}$ of $\Gamma_{B_{i}^{(l)}}$ determined by $T_{i}^{(l)}$.

(v) For each $j \in\{1, \ldots, n\}$, there exists a tilted algebra $B_{j}^{(r)}=\operatorname{End}_{H_{j}^{(r)}}\left(T_{j}^{(r)}\right)$, where $H_{j}^{(r)}$ is the path algebra $K \Delta_{j}^{(r)}, T_{j}^{(r)}$ is a tilting $H_{j}^{(r)}$-module without nonzero postprojective direct summands, $B_{j}^{(r)}$ is a quotient algebra of $B$, and $\mathscr{D}_{j}^{(r)}$ coincides 
with the torsion part $\mathscr{X}\left(T_{j}^{(r)}\right) \cap \mathscr{C}_{T_{j}^{(r)}}$ of the connecting component $\mathscr{C}_{T_{j}^{(r)}}$ of $\Gamma_{B_{j}^{(r)}}$ determined by $T_{j}^{(r)}$.

(vi) $\mathscr{Y}(T)=\operatorname{add}\left(\mathscr{Y}\left(T_{1}^{(l)}\right) \cup \cdots \cup \mathscr{Y}\left(T_{m}^{(l)}\right) \cup\left(\mathscr{Y}(T) \cap \mathscr{C}_{T}\right)\right)$.

(vii) $\mathscr{X}(T)=\operatorname{add}\left(\left(\mathscr{X}(T) \cap \mathscr{C}_{T}\right) \cup \mathscr{X}\left(T_{1}^{(r)}\right) \cup \cdots \cup \mathscr{X}\left(T_{n}^{(r)}\right)\right)$.

(viii) The Auslander-Reiten quiver $\Gamma_{B}$ of $B$ has the disjoint union decomposition

$$
\Gamma_{B}=\left(\bigcup_{i=1}^{m} \mathscr{Y} \Gamma_{B_{i}^{(l)}}\right) \cup \mathscr{C}_{T} \cup\left(\bigcup_{j=1}^{n} \mathscr{X} \Gamma_{B_{j}^{(r)}}\right),
$$

where

(a) For each $i \in\{1, \ldots, m\}$, $\mathscr{Y} \Gamma_{B_{i}^{(l)}}$ is the union of all components of $\Gamma_{B_{i}^{(l)}}$ contained entirely in $\mathscr{Y}\left(T_{i}^{(l)}\right)$, and hence consists of a unique postprojective component $\mathscr{P}^{B_{i}^{(l)}}$ and a $\mathbb{P}_{1}(K)$-family $\mathscr{T}_{i}^{B_{i}^{(l)}}=\left(\mathscr{T}_{\lambda}^{B_{i}^{(l)}}\right)_{\lambda \in \mathbb{P}_{1}(K)}$ of pairwise orthogonal generalized standard ray tubes;

(b) For each $j \in\{1, \ldots, n\}, \mathscr{X} \Gamma_{B_{j}^{(r)}}$ is the union of all components of $\Gamma_{B_{j}^{(r)}}$ contained entirely in $\mathscr{X}\left(T_{j}^{(r)}\right)$, and hence consists of a unique preinjective component $\mathscr{Q}^{B_{j}^{(r)}}$ and a $\mathbb{P}_{1}(K)$-family $\mathscr{T}^{B_{j}^{(r)}}=\left(\mathscr{T}_{\lambda}^{B_{j}^{(r)}}\right)_{\lambda \in \mathbb{P}_{1}(K)}$ of pairwise orthogonal generalized standard coray tubes.

The following theorem follows from [18](part (i)) and [65, p.376](parts (ii) and (iii)).

Theorem 5.3. Let $A$ be a cycle-finite algebra, $X$ a directing module in $\bmod A$, and $B=\operatorname{supp} X$. Then the following statements hold:

(i) $B$ is a convex subcategory of $A$.

(ii) $B$ is a tame tilted algebra.

(iii) $X$ belongs to a connecting component of $\Gamma_{B}$.

We refer to [17] and [29] (respectively, [55] and [56]) for a classification of representation-finite (respectively, tame representation-infinite) tame tilted algebras with sincere directing modules.

The class of tilted algebras was extended in [62] to the class of double tilted algebras, and next in [63] to the class of generalized double tilted algebras, containing the class of all algebras of finite type, by extending the concept of a section to the concept of a multisection.

Following [63], a full connected subquiver $\Delta$ of a component $\mathscr{C}$ of the AuslanderReiten quiver $\Gamma_{A}$ of an algebra $A$ is said to be a multisection if the following conditions are satisfied:

(i) $\Delta$ is almost acyclic.

(ii) $\Delta$ is convex in $\mathscr{C}$. 
(iii) For each $\tau_{A}$-orbit $\mathscr{O}$ in $\mathscr{C}$, we have $1 \leq|\Delta \cap \mathscr{O}|<\infty$.

(iv) $|\Delta \cap \mathscr{O}|=1$ for all but finitely many $\tau_{A}$-orbits $\mathscr{O}$ in $\mathscr{C}$.

(v) No proper full convex subquiver of $\Delta$ satisfies (i)-(iv).

It has been proved in [63, Theorem 2.5] that a component $\mathscr{C}$ of $\Gamma_{A}$ is almost acyclic if and only if $\mathscr{C}$ admits a multisection $\Delta$. Moreover, for a multisection $\Delta$ of a component $\mathscr{C}$ of $\Gamma_{A}$, the following full subquivers of $\mathscr{C}$ were defined in [63]:

(i) $\Delta_{l}^{\prime}=\{X \in \Delta$; there is a nonsectional path in $\mathscr{C}$ from $X$ to a projective module $P\}$,

(ii) $\Delta_{r}^{\prime}=\{X \in \Delta$; there is a nonsectional path in $\mathscr{C}$ from an injective module $I$ to $X\}$,

(iii) $\Delta_{l}^{\prime \prime}=\left\{X \in \Delta_{l}^{\prime} ; \tau_{A}^{-1} X \notin \Delta_{l}^{\prime}\right\}, \quad \Delta_{r}^{\prime \prime}=\left\{X \in \Delta_{r}^{\prime} ; \tau_{A} X \notin \Delta_{r}^{\prime}\right\}$,

(iv) $\Delta_{l}=\left(\Delta \backslash \Delta_{r}^{\prime}\right) \cup \tau_{A} \Delta_{r}^{\prime \prime}, \quad \Delta_{c}=\Delta_{l}^{\prime} \cap \Delta_{r}^{\prime}, \quad \Delta_{r}=\left(\Delta \backslash \Delta_{l}^{\prime}\right) \cup \tau_{A}^{-1} \Delta_{l}^{\prime \prime}$.

Then $\Delta_{l}$ is called the left part of $\Delta, \Delta_{r}$ the right part of $\Delta$, and $\Delta_{c}$ the core of $\Delta$.

Lemma 5.4. Let $A$ be an algebra, $\mathscr{C}$ a component of $\Gamma_{A}$ and $\Delta$ a multisection of $\mathscr{C}$. The following statements hold

(i) Every cycle of $\mathscr{C}$ lies in $\Delta_{c}$.

(ii) $\Delta_{c}$ is finite.

(iii) Every indecomposable module $X$ in $\mathscr{C}$ is in $\Delta_{c}$, or a predecessor of $\Delta_{l}$ or a successor of $\Delta_{r}$ in $\mathscr{C}$.

(iv) $\Delta$ is faithful if and only if $\mathscr{C}$ is faithful.

Moreover, in [63] a numerical invariant $w(\Delta) \in \mathbb{N} \cup\{\infty\}$ of a multisection $\Delta$ of $\mathscr{C}$, called the width of $\Delta$, was introduced such that $\mathscr{C}$ is acyclic if and only if $w(\Delta)<\infty$, and $w(\Delta)=1$ if and only if $\Delta$ is a section.

The following facts proved in [63, Proposition 2.11] show that the core and the width of a multisection of an almost cyclic component $\mathscr{C}$ are uniquely determined by $\mathscr{C}$.

Proposition 5.5. Let $A$ be an algebra, $\mathscr{C}$ a component of $\Gamma_{A}$ and $\Delta, \Sigma$ multisections of $\mathscr{C}$. Then $\Delta_{c}=\Sigma_{c}$ and $w(\Delta)=w(\Sigma)$.

Following [63], an algebra $B$ is said to be a generalized double tilted algebra if the following conditions are satisfied:

(1) $\Gamma_{B}$ admits a component $\mathscr{C}$ with a faithful multisection $\Delta$.

(2) There exists a tilted quotient algebra $B^{(l)}$ of $B$ (not necessarily connected) such that $\Delta_{l}$ is a disjoint union of sections of the connecting components of the connected parts of $B^{(l)}$ and the category of all predecessors of $\Delta_{l}$ in ind $B$ coincides with the category of all predecessors of $\Delta_{l}$ in ind $B^{(l)}$.

(3) There exists a tilted quotient algebra $B^{(r)}$ of $B$ (not necessarily connected) such that $\Delta_{r}$ is a disjoint union of sections of the connecting components of the connected parts of $B^{(r)}$, and the category of all successors of $\Delta_{r}$ in ind $B$ coincides with the category of all successors of $\Delta_{r}$ in ind $B^{(r)}$.

Then $B^{(l)}$ is called a left tilted part of $B$ and $B^{(r)}$ a right tilted part of $B$.

The following generalization of Theorem 5.1 proved in [63, Theorem 3.1], gives a handy criterion for an algebra to be a generalized double tilted algebra. 
Theorem 5.6. Let $B$ be an algebra. The following conditions are equivalent:

(i) $B$ is a generalized double tilted algebra.

(ii) The quiver $\Gamma_{B}$ admits a component $\mathscr{C}$ with a faithful multisection $\Delta$ such that $\operatorname{Hom}_{B}\left(U, \tau_{B} V\right)=0$, for all modules $U \in \Delta_{r}$ and $V \in \Delta_{l}$.

(iii) The quiver $\Gamma_{B}$ admits a faithful generalized standard almost acyclic component $\mathscr{C}$.

In particular, we obtain the following characterization of tame generalized double tilted algebras.

Theorem 5.7. Let $B$ be a generalized double tilted algebra, $\mathscr{C}$ a faithful generalized standard almost cyclic component of $\Gamma_{B}$, and $\Delta$ a multisection of $\mathscr{C}$. The following conditions are equivalent:

(i) $B$ is tame.

(ii) $B$ is cycle-finite.

(iii) $\Delta_{l}$ and $\Delta_{r}$ are disjoint unions of Euclidean quivers.

(iv) The tilted algebras $B^{(l)}$ and $B^{(r)}$ are tame.

(v) The Auslander-Reiten quiver $\Gamma_{B}$ of $B$ has disjoint union decomposition

$$
\Gamma_{B}=\mathscr{Y} \Gamma_{B^{(l)}} \cup \mathscr{C} \cup \mathscr{X} \Gamma_{B^{(r)}},
$$

where

(a) $\mathscr{Y} \Gamma_{B^{(l)}}$ is the union of all connected components of $\Gamma_{B^{(l)}}$ contained entirely in the torsion-free part $\mathscr{Y}\left(B^{(l)}\right)$, and $\mathscr{Y} \Gamma_{B^{(l)}}$ is a disjoint union of postprojective components of Euclidean type and $\mathbb{P}_{1}(K)$-families of pairwise orthogonal generalized standard ray tubes.

(b) $\mathscr{X} \Gamma_{B^{(r)}}$ is the union of all connected components of $\Gamma_{B^{(r)}}$ contained entirely in the torsion part $\mathscr{X}\left(B^{(r)}\right)$, and $\mathscr{X} \Gamma_{B^{(r)}}$ is a disjoint union of preinjective components of Euclidean type and $\mathbb{P}_{1}(K)$-families of pairwise orthogonal generalized standard coray tubes.

We end this section with an example of a tame generalized double tilted algebra, illustrating the above considerations.

Example 5.8. Let $B=K Q / I$, where $Q$ is the quiver

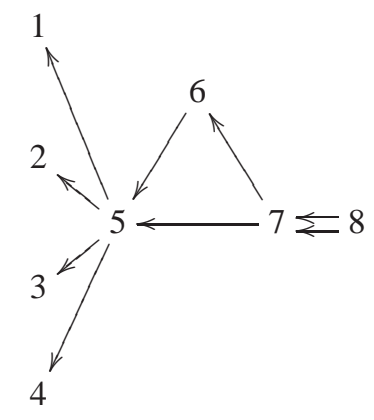


and $I$ is the ideal of $K Q$ generated by all paths of $Q$ of length 2 (see [63, Example 4.3]). Then $B$ is a tame generalized double tilted algebra of global dimension 4 and $\Gamma_{B}$ admits a generalized standard component $\mathscr{C}$ of the form

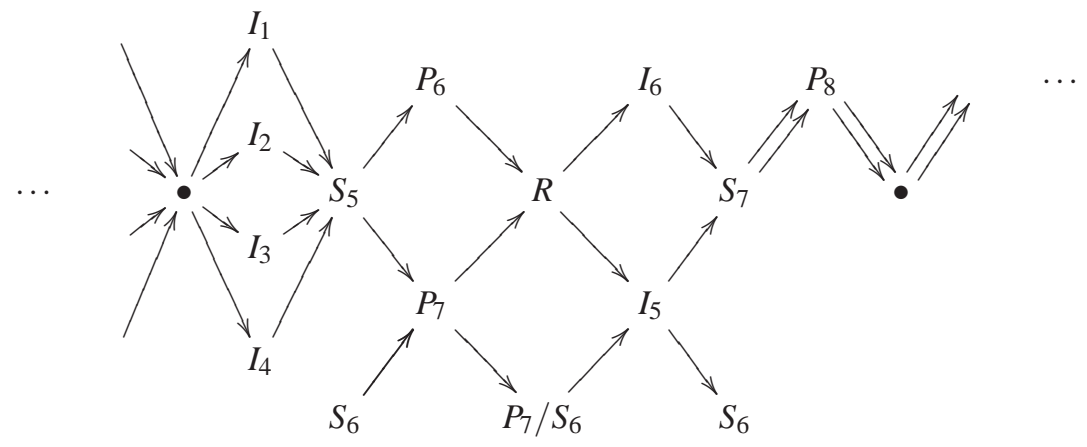

with a faithful multisection $\Delta$ formed by the indecomposable injective modules $I_{1}, I_{2}, I_{3}, I_{4}, I_{5}, I_{6}$ (at the vertices $1,2,3,4,5,6$ ), the indecomposable projective modules $P_{6}, P_{7}, P_{8}$ (at the vertices $6,7,8$ ), the simple modules $S_{5}, S_{6}, S_{7}$ (at the vertices 5 , $6,7)$ and the modules $P_{7} / S_{6}, R=\tau_{B}^{-1} S_{5}=\tau_{B} S_{7}$. Then the left part $\Delta_{l}$ of $\Delta$ consists of the modules $I_{1}, I_{2}, I_{3}, I_{4}, S_{5}, P_{6}$ and is a section of the preinjective connecting component of the tame tilted algebra $B^{(l)}$ being the convex subcategory of $B$ given by the vertices $1,2,3,4,5,6$. The right part $\Delta_{r}$ of $\Delta$ consists of the modules $I_{6}, S_{7}, P_{8}$ and is a section of the postprojective connecting component of the tame tilted algebra $B^{(r)}$ being the convex subcategory of $B$ given by the vertices $6,7,8$. Moreover, the core $\Delta_{c}$ of $\Delta$ consists of the modules $S_{6}, P_{7}, P_{7} / S_{6}, R, I_{5}$ and is the cyclic part of the Auslander-Reiten quiver $\Gamma_{B^{(c)}}$ of the representation-finite convex subcategory $B^{(c)}=\operatorname{supp} \Delta_{c}$ of $B$ given by the vertices $5,6,7$. We also note that every module in $\operatorname{ind} B$ belongs to one of its full subcategories ind $B^{(l)}$, ind $B^{(c)}$, or ind $B^{(r)}$.

\section{Cyclic components of cycle-finite algebras}

Let $A$ be an algebra. We denote by ${ }_{c} \Gamma_{A}$ the translation subquiver of $\Gamma_{A}$, called the cyclic part of $\Gamma_{A}$, obtained by removing from $\Gamma_{A}$ all acyclic modules and the arrows attached to them. The connected components of ${ }_{c} \Gamma_{A}$ are said to be cyclic components of $\Gamma_{A}$ (see [50]). The following result from [50, Proposition 5.1] will be very useful.

Proposition 6.1. Let $A$ be an algebra and $X, Y$ be two cyclic modules of $\Gamma_{A}$. Then $X$ and $Y$ belong to the same cyclic component of $\Gamma_{A}$ if and only if there is an oriented cycle in $\Gamma_{A}$ passing through $X$ and $Y$.

Moreover, we have the following property of the support algebras of cyclic components of the Auslander-Reiten quivers of cycle-finite algebras (see [49]). 
Proposition 6.2. Let $A$ be a cycle-finite algebra, $\Gamma$ a cyclic component of $\Gamma_{A}$ and $B=\operatorname{supp} \Gamma$. Then $B$ is a convex subcategory of $A$.

Let $A$ be an algebra and $\mathscr{C}$ be a component of $\Gamma_{A}$. We denote by ${ }_{l} \mathscr{C}$ the left stable part of $\mathscr{C}$, obtained by removing from $\mathscr{C}$ the $\tau_{A}$-orbits containing projective modules, and by ${ }_{r} \mathscr{C}$ the right stable part of $\mathscr{C}$, obtained by removing from $\mathscr{C}$ the $\tau_{A}$-orbits containing injective modules. We note that if $\mathscr{C}$ is an infinite component of $\Gamma_{A}$ then ${ }_{l} \mathscr{C}$ or ${ }_{r} \mathscr{C}$ is not empty.

The following theorem from [49, Theorem 1] describes the supports of infinite cyclic components of the Auslander-Reiten quivers of cycle-finite algebras.

Theorem 6.3. Let $A$ be a cycle-finite algebra and $\Gamma$ an infinite cyclic component of $\Gamma_{A}$. Then there exist infinite full translation subquivers $\Gamma_{1}, \ldots, \Gamma_{r}$ of $\Gamma$ such that the following statements hold.

(i) For each $i \in\{1, \ldots, r\}, \Gamma_{i}$ is a cyclic coherent full translation subquiver of $\Gamma_{A}$.

(ii) For each $i \in\{1, \ldots, r\}, B^{(i)}=\operatorname{supp} \Gamma_{i}$ is a tame generalized multicoil algebra and a quotient algebra of $A$.

(iii) $\Gamma_{1}, \ldots, \Gamma_{r}$ are pairwise disjoint full translation subquivers of $\Gamma$ and $\Gamma^{c c}=\Gamma_{1} \cup$ $\ldots \cup \Gamma_{r}$ is a maximal cyclic coherent and cofinite full translation subquiver of $\Gamma$.

(iv) $B\left(\Gamma \backslash \Gamma^{c c}\right)=A / \operatorname{ann}_{A}\left(\Gamma \backslash \Gamma^{c c}\right)$ is of finite representation type.

The following example illustrates the above theorem.

Example 6.4. Let $A=K Q / I$, where $Q$ is the quiver

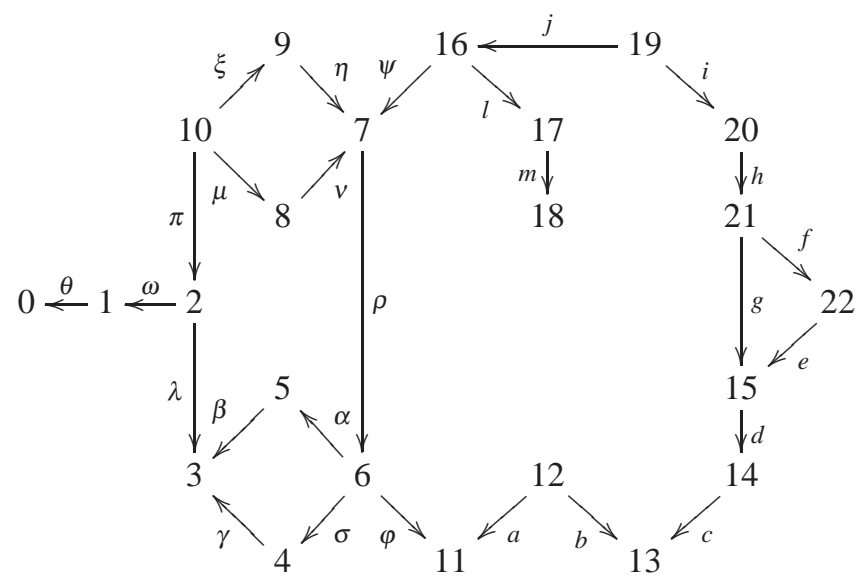

and $I$ is the ideal in the path algebra $K Q$ of $Q$ generated by the elements $\alpha \beta-\sigma \gamma$, $\xi \eta-\mu \nu, \pi \lambda-\xi \eta \rho \alpha \beta, \rho \varphi, \psi \rho, j l, d c, e d, g d, h g, h f$, ih. Then $A$ is a cycle-finite and $\Gamma_{A}$ admits a component $\mathscr{C}$ of the form 


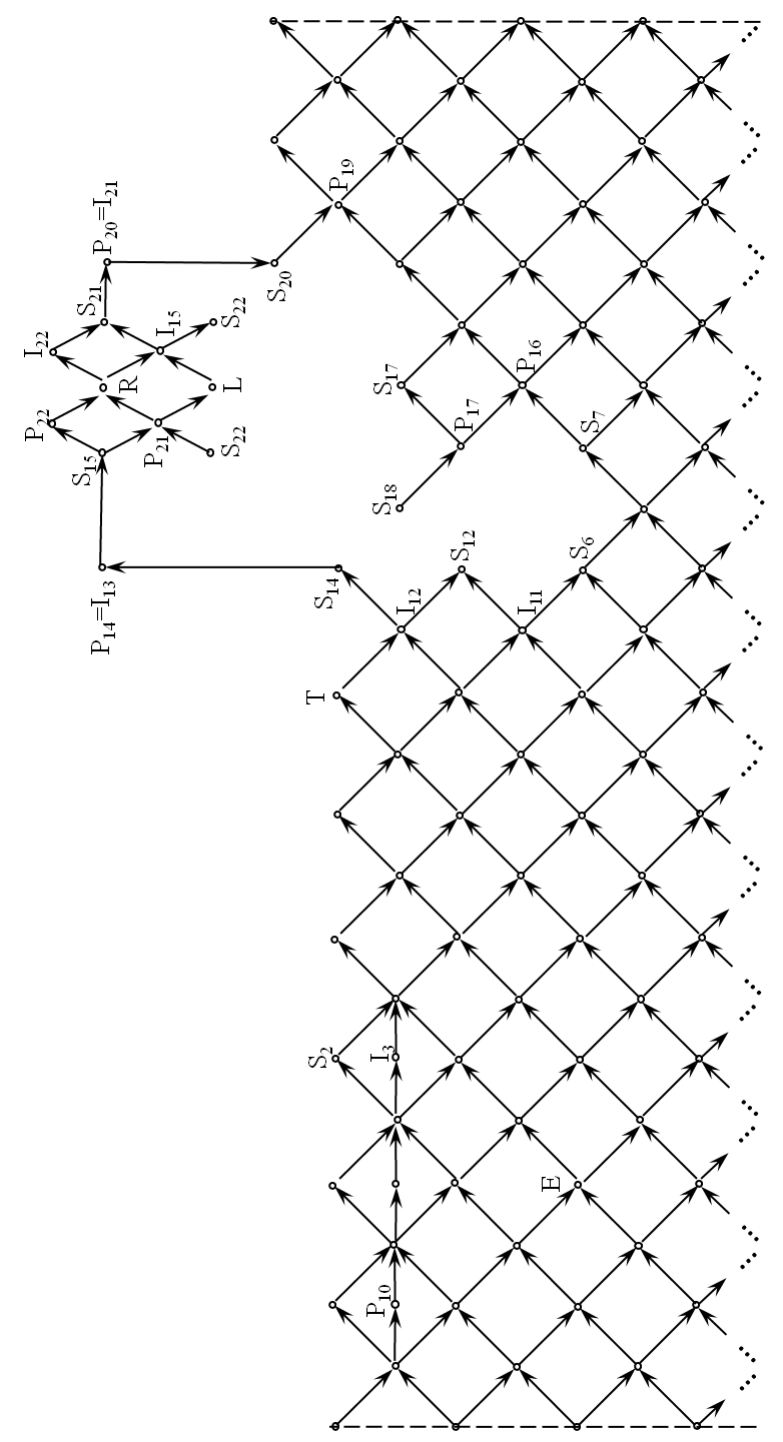

The cyclic part $\Gamma$ of $\mathscr{C}$ is obtain by removing from $\mathscr{C}$ the (directing) modules $S_{12}, S_{17}, S_{18}, P_{17}$ and the arrows attached to them. The maximal cyclic coherent part $\Gamma^{c c}$ of $\Gamma$ is the full translation subquiver of $\mathscr{C}$ obtained by removing the modules $S_{12}, I_{12}, T, S_{14}, P_{14}=I_{13}, S_{15}, P_{21}, S_{22}, L, P_{22}, R, I_{15}, I_{22}, S_{21}, P_{20}=I_{21}, S_{20}, S_{17}, P_{17}$, $S_{18}$ and the arrows attached to them. Further, $\Gamma^{c c}$ is the cyclic part of the maximal almost cyclic coherent full translation subquiver $\Gamma^{*}$ of $\mathscr{C}$ obtained by removing the modules $P_{14}=I_{13}, S_{15}, P_{21}, S_{22}, L, P_{22}, R, I_{15}, I_{22}, S_{21}, P_{20}=I_{21}$ and the arrows attached to them.

Let $B=A / \operatorname{ann} \Gamma$. Then $B=A / \operatorname{ann} \Gamma^{*}$, because ann $\Gamma=\operatorname{ann} \Gamma^{*}$. Observe that $B=K Q_{B} / I_{B}$, where $Q_{B}$ is the full subquiver of $Q$ given by all vertices of $Q$ except 
$15,21,22$, and $I_{B}=I \cap K Q_{B}$. We claim that $B$ is a tame generalized multicoil algebra. Consider the path algebra $C=K \Delta$ of the full subquiver of $Q$ given by the vertices $4,5,6,7,8,9$. Then $C$ is a hereditary algebra of Euclidean type $\widetilde{\mathbb{D}}_{5}$, and hence a tame concealed algebra. It is know that $\Gamma_{C}$ admits $\mathbb{P}_{1}(K)$-family $\mathscr{T}_{\lambda}^{C}, \lambda \in \mathbb{P}_{1}(K)$, of pairwise orthogonal generalized standard stable tubes, having a unique stable tube $\mathscr{T}_{\lambda}$ of rank 3 with the mouth formed by the modules $S_{6}=\tau_{C} S_{7}, S_{7}=\tau_{C} E$, $E=\tau_{C} S_{6}$, where $E$ is the unique indecomposable $C$-module with the dimension vector $\operatorname{dim} E=\frac{{ }_{1}^{1}}{1}{ }_{1}$, , (see [67, Theorem XIII 2.9]).

Then $B$ is the generalized multicoil enlargement of $C$, obtained by applications

- two admissible operations of types (ad $1^{*}$ ) with the pivots $S_{6}$ and $S_{12}$, creating the vertices 11,12, 13 and the arrows $\varphi, a, b, c$;

- two admissible operations of types $\left(\operatorname{ad~} 1^{*}\right)$ with the pivots $E$ and $S_{2}$, creating the vertices $3,2,1,0$ and the arrows $\beta, \gamma, \lambda, \omega, \theta$;

- two admissible operations of types (ad 1$)$ with the pivots $S_{7}$ and $S_{16}$, creating the vertices $16,17,18,19,20$ and the arrows $\psi, l, m, j, i$;

- one admissible operation of type (ad 3 ) with the pivot the radical of $P_{10}$, creating the vertex 10 and the arrows $\xi, \mu, \pi$.

Then the left part $B^{(l)}$ of $B$ is the convex subcategory of $B$ (and of $A$ ) given by the vertices $0,1,2,3,4,5,6,7,8,9,11,12,13,14$, and is a tilted algebra of Euclidean type $\widetilde{\mathbb{D}}_{13}$ with the connecting postprojective component $\mathscr{P} B^{(l)}$ containing all indecomposable projective $B^{(l)}$-modules. The right part $B^{(r)}$ of $B$ is the convex subcategory of $B$ (and of $A$ ) given by the vertices 4, 5, 6, 7, 8, 9, 16, 17, 18, 19, 20, and is a tilted algebra of Euclidean type $\widetilde{\mathbb{D}}_{10}$ with the connecting preinjective component $\mathscr{Q}^{B^{(r)}}$ containing all indecomposable injective $B^{(r)}$-modules. We also note that the algebra $B\left(\Gamma \backslash \Gamma^{c c}\right)=A / \operatorname{ann}_{A}\left(\Gamma \backslash \Gamma^{c c}\right)$ is the representation-finite convex subcategory of $A$ given by the vertices $12,13,14,15,20,21,22$. It follows from Theorem 4.8 that the Auslander-Reiten quiver $\Gamma_{B}$ of the generalized multicoil enlargement $B$ of $C$ is of the form

$$
\Gamma_{B}=\mathscr{P}^{B} \cup \mathscr{C}^{B} \cup \mathscr{Q}^{B},
$$

where $\mathscr{P}^{B}=\mathscr{P}^{B^{(l)}}, \mathscr{Q}^{B}=\mathscr{Q}^{B^{(r)}}$, and $\mathscr{C}^{B}$ is the $\mathbb{P}_{1}(K)$-family $\mathscr{C}_{\lambda}^{B}, \lambda \in \mathbb{P}_{1}(K)$, of pairwise orthogonal generalized multicoils such that $\mathscr{C}_{1}^{B}=\Gamma^{*}$ and $\mathscr{C}_{\lambda}^{B}=\mathscr{T}_{\lambda}^{C}$ for all $\lambda \in \mathbb{P}_{1}(K) \backslash\{1\}$. Hence $\Gamma_{A}$ is of the form

$$
\Gamma_{A}=\mathscr{P}^{A} \cup \mathscr{C}^{A} \cup \mathscr{Q}^{A},
$$

where $\mathscr{P}^{A}=\mathscr{P}^{B^{(l)}}, \mathscr{Q}^{A}=\mathscr{Q}^{B^{(r)}}$, and $\mathscr{C}^{A}$ is the $\mathbb{P}_{1}(K)$-family $\mathscr{C}_{\lambda}^{A}, \lambda \in \mathbb{P}_{1}(K)$, of pairwise orthogonal generalized standard components such that $\mathscr{C}_{1}^{A}=\mathscr{C}, \mathscr{C}_{\lambda}^{A}=\mathscr{T}_{\lambda}^{C}$ for all $\lambda \in \mathbb{P}_{1}(K) \backslash\{1\}$. Moreover, we have

$$
\operatorname{Hom}_{A}\left(\mathscr{C}^{A}, \mathscr{P}^{A}\right)=0, \operatorname{Hom}_{A}\left(\mathscr{Q}^{A}, \mathscr{C}^{A}\right)=0, \operatorname{Hom}_{A}\left(\mathscr{Q}^{A}, \mathscr{P}^{A}\right)=0 .
$$


In particular, $A$ is a cycle-finite algebra with $\left(\operatorname{rad}^{\infty}(\bmod A)\right)^{3}=0$.

The following theorem from [49, Theorem 2] describes the supports of finite cyclic components of the Auslander-Reiten quivers of cycle-finite algebras.

Theorem 6.5. Let $A$ be a cycle-finite algebra and $\Gamma$ a finite cyclic component of $\Gamma_{A}$. Then the following statements hold.

(i) $B=\operatorname{supp} \Gamma$ is a tame generalized double tilted algebra.

(ii) $\Gamma$ is the core of the connecting component $\mathscr{C}_{B}$ of $\Gamma_{B}$.

We note that if the core of an almost cyclic component of an Auslander-Reiten quiver $\Gamma_{A}$ is not empty that it contains a projective module and an injective module. Then we obtain the following consequence of Theorem 6.5 .

Corollary 6.6. Let $A$ be a cycle-finite algebra. Then the number of finite cyclic components of $\Gamma_{A}$ is bounded by the rank of $K_{0}(A)$.

Observe also that for a cycle-finite algebra $A$ of infinite representation type there are infinitely many (infinite) cyclic components of $\Gamma_{A}$, since $\Gamma_{A}$ contains infinitely many stable tubes (see Theorems 3.1 and 3.3).

\section{The structure theorems}

Let $A$ be a cycle-finite algebra and $X$ a module in ind $A$. Then $X$ is a directing module if and only if $X$ is an acyclic vertex of $\Gamma_{A}$. Hence $X$ is nondirecting if and only if $X$ belongs to a cyclic component of $\Gamma_{A}$. Then the following structure theorem is a direct consequence of Theorems 5.3 6.3 and Propositions 6.1, 6.2

Theorem 7.1. Let $A$ be a cycle-finite algebra. Then there exist quotient algebras $B_{1}, \ldots, B_{p}$ of $A$ such that the following statements hold.

(i) For each $i \in\{1, \ldots, p\}, B_{i}$ is either a tame generalized multicoil algebra or a tame generalized double tilted algebra.

(ii) ind $A=\bigcup_{i=1}^{p}$ ind $B_{i}$.

It follows from Theorem 5.2 that for a tame tilted algebra $B$, all but finitely many modules in ind $B$ are indecomposable modules over the left tilted algebras $B_{1}^{(l)}, \ldots, B_{m}^{(l)}$ of Euclidean types or over the right tilted algebras $B_{1}^{(r)}, \ldots, B_{n}^{(r)}$ of Euclidean types. Moreover, all representation-infinite tilted algebras of Euclidean types and all tubular algebras are tame generalized multicoil algebras. Therefore, applying Theorem 4.8 we obtain the following completion to Theorem 7.1.

Theorem 7.2. Let $A$ be a cycle-finite algebra. Then there exist tame generalized multicoil quotient algebras $B_{1}, \ldots, B_{q}$ of $A$ whose indecomposable finite dimensional modules exhaust all but finitely many isoclasses of modules in ind $A$.

Moreover, we have the following information on nondirecting indecomposable modules over cycle-finite algebras. 
Theorem 7.3. Let $A$ be a cycle-finite algebra. Then there exist tame generalized multicoil quotient algebras $B_{1}, \ldots, B_{q}$ of $A$ such that all but finitely many isomorphism classes of nondirecting modules in ind $A$ belong to generalized multicoils of the Auslander-Reiten quivers $\Gamma_{B_{1}}, \ldots, \Gamma_{B_{q}}$ of $B_{1}, \ldots, B_{q}$.

We exhibit an example of a cycle-finite algebra having a nongeneralized standard Auslander-Reiten component.

Example 7.4. Let $Q$ be the quiver

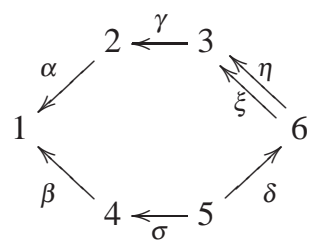

$I$ the ideal in the path algebra $K Q$ of $Q$ generated by the elements $\gamma \alpha, \sigma \beta, \eta \gamma, \xi \gamma$, $\delta \xi, \delta \eta$, and $A=K Q / I$ the associated bound quiver algebra. Then $A$ is a cycle-finite algebra whose Auslander-Reiten quiver $\Gamma_{A}$ has the disjoint union form

$$
\Gamma_{A}=\mathscr{C} \cup\left(\bigcup_{\lambda \in \mathbb{P}_{1}(K)} \mathscr{T}_{\lambda}\right)
$$

where $\mathscr{T}_{\lambda}, \lambda \in \mathbb{P}_{1}(K)$, is the family of stable tubes of rank 1 over the Kronecker path algebra $H=K \Delta$ given by the subquiver $\Delta$ of $Q$ formed by the arrows $\xi$ and $\eta$, and $\mathscr{C}$ is the following glueing of the preprojective component $\mathscr{P}(H)$ and the preinjective component $\mathscr{Q}(H)$ of $\Gamma_{A}$ into a component of $\Gamma_{A}$

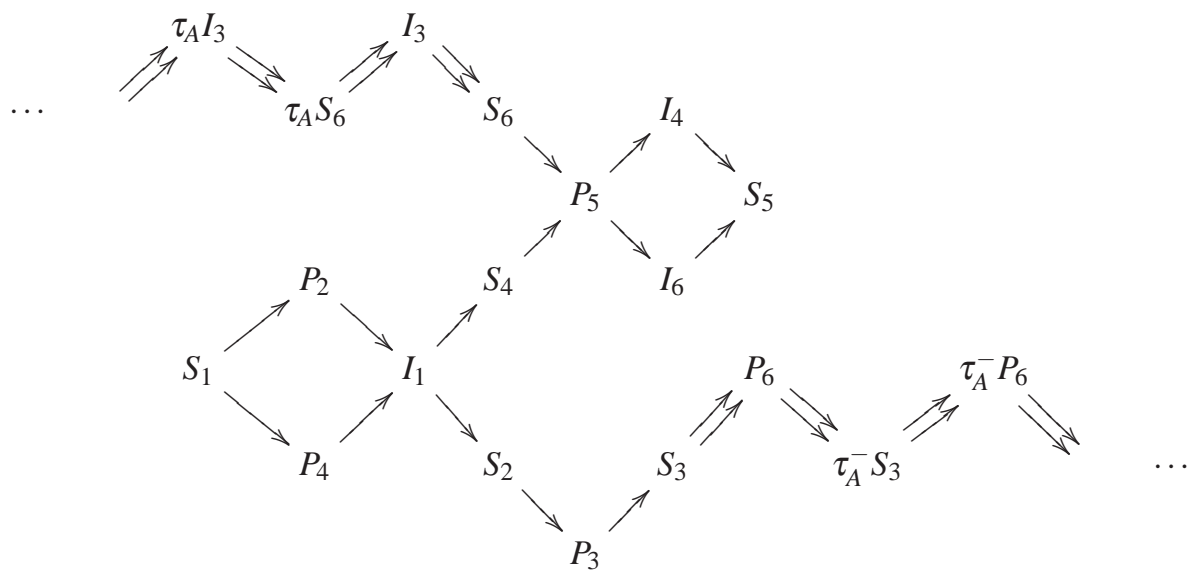

Observe that the simple module $S_{3}$ is the socle of the injective module $I_{3}$ and the canonical monomorphism $S_{3} \rightarrow I_{3}$ belongs to $\operatorname{rad}^{\infty}(\bmod A)$, because it factors through any stable tube $\mathscr{T}_{\lambda}$. Hence $\mathscr{C}$ is not generalized standard. On the other hand, every cycle in ind $A$ consists of modules of a stable tube $\mathscr{T}_{\lambda}$ and hence is finite, be- 
cause the stable tubes $\mathscr{T}_{\lambda}$ are generalized standard. Therefore, $A$ is a cycle-finite algebra.

We end this section with an example of an algebra $A$ with partially ordered Auslander-Reiten components of $\Gamma_{A}$ which is not cycle-finite.

Example 7.5. Let $Q$ be the quiver

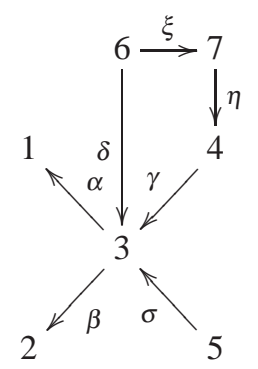

$I$ the ideal in the path algebra $K Q$ of $Q$ generated by the elements $\delta \alpha, \delta \beta, \xi \eta$ and $\eta \gamma$, and $A=K Q / I$ the associated bound quiver algebra. Then $A$ is a tame algebra whose Auslander-Reiten quiver $\Gamma_{A}$ has the disjoint union form

$$
\Gamma_{A}=\mathscr{P}(A) \cup \mathscr{T}^{A} \cup \mathscr{C},
$$

where $\mathscr{P}(A)$ is a preprojective component, $\mathscr{T}^{A}=\left(\mathscr{T}_{\lambda}^{A}\right)_{\lambda \in \mathbb{P}_{1}(K) \backslash\{1\}}$ is a family of pairwise orthogonal generalized standard stable tubes, and $\mathscr{C}$ is a component of the form below

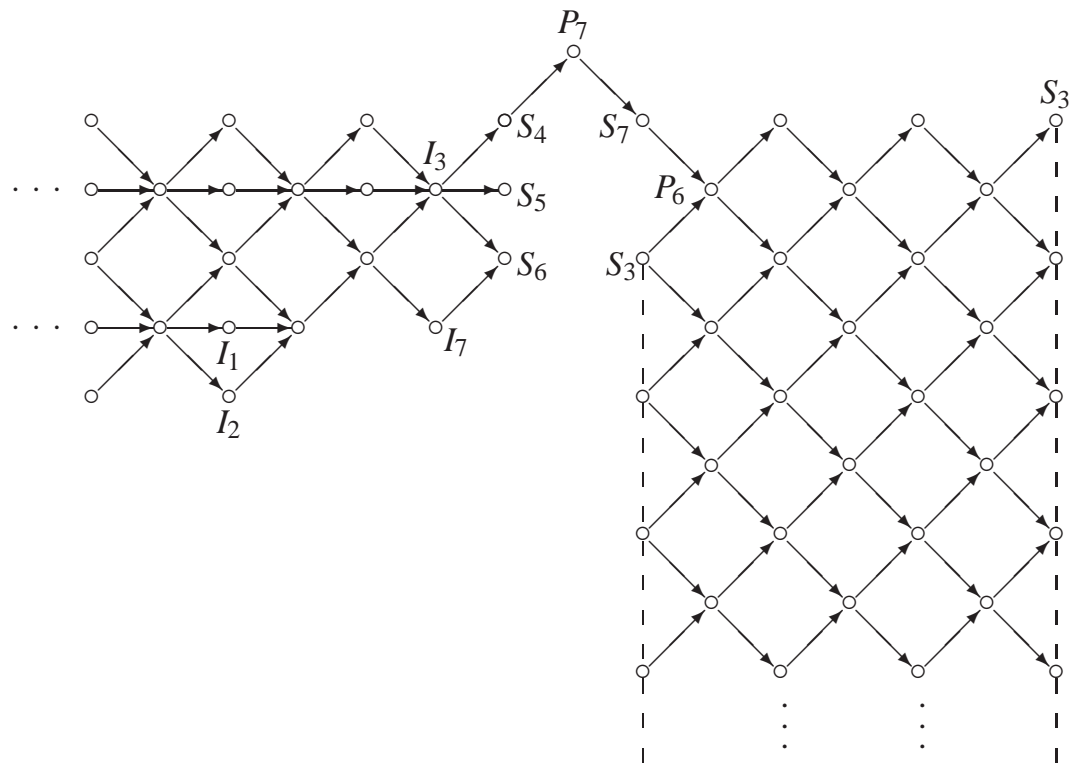


(see [36, Example2.6]). Then we obtain that the components of $\Gamma_{A}$ are partially ordered in ind $A$. In particular, we conclude that for every cycle $X_{0} \rightarrow X_{1} \rightarrow \cdots \rightarrow$ $X_{r-1} \rightarrow X_{r}=X_{0}$ in ind $A$, all modules $X_{0}, X_{1}, \ldots, X_{r-1}$ belong to the same component of $\Gamma_{A}$. On the other hand, we have in ind $A$ a cycle

$$
I_{3} \stackrel{f_{1}}{\longrightarrow} P_{7} \stackrel{f_{2}}{\longrightarrow} P_{6} \stackrel{f_{3}}{\longrightarrow} I_{3}
$$

consisting of modules from $\mathscr{C}$ and with $f_{3}$ in $\operatorname{rad}^{\infty}(\bmod A)$, because there is no path in $\mathscr{C}$ from $P_{6}$ to $I_{3}$. Therefore, $A$ is not a cycle-finite algebra.

\section{Discrete indecomposable modules}

The aim of this section is to establish a common bound on the number of discrete indecomposable modules of a fixed dimension vector over a cycle-finite algebra.

Lemma 8.1. Let $A$ be an algebra, $\Gamma$ a generalized standard generalized multicoil of $\Gamma_{A}, n$ the rank of $K_{0}(A)$, and $\mathbf{d}$ a nonnegative vector of $K_{0}(A)$. Then the number of indecomposable modules $X$ in $\Gamma$ with $\operatorname{dim} X=\mathbf{d}$ is bounded by $n$. Moreover, if $\Gamma$ consists of modules which do not lie on infinite cycles then the number of indecomposable modules $X$ in $\Gamma$ with $\operatorname{dim} X=\mathbf{d}$ is bounded by $n-1$.

Proof. Without loss of generality we may assume that $A$ is the support algebra of $\Gamma$. Let $\Gamma$ be an arbitrary generalized multicoil of $\Gamma_{A}$ which is generalized standard. We shall prove our claim by induction on the number $m$ of admissible operations which we have to do on a finite family $\mathscr{T}_{1}, \mathscr{T}_{2}, \ldots, \mathscr{T}_{S}$ of generalized standard stable tubes in order to obtain the generalized multicoil $\Gamma$. If $m=1$, then we can only do the admissible operation (ad 1) or (ad $\left.1^{*}\right)$, so $s=1$. In this case our statement follows from [46, Proposition 4.1].

Let $m>1$. If the $m$ th admissible operation is of type ( $\operatorname{ad} 1)$, then by definition of (ad 1) we have $\operatorname{dim} V=\operatorname{dim} W$ for any modules $V, W \in\left\{Z_{i j}, X_{i}^{\prime}\right\}, i \geq 0,1 \leq j \leq t$ such that $V \neq W$. Therefore, the number of indecomposable modules with the same dimension vector does not change. If it is of type $\left(\mathrm{ad} 1^{*}\right)$ then the situation is the same. If the $m$ th admissible operation is of type ( $\operatorname{ad} 2)$, then in the sequence of earlier $m-1$ admissible operations, there is an operation of type $\left(\operatorname{ad~} 1^{*}\right)$ or $\left(\operatorname{ad~} 5^{*}\right)$ which contains an operation $\left(\mathrm{fad} 1^{*}\right)$ which gives rise to the pivot $X$ of $(\operatorname{ad} 2)$, and the operations done between these two must not affect the support of $\operatorname{Hom}_{A}(X,-)$ restricted to the generalized multicoil containing $X$. Let $t$ be the parameter of such operation $\left(\operatorname{ad~} 1^{*}\right)$ or like in definition of $\left(\right.$ fad $\left.1^{*}\right)$. Note that in general, in the sequence of earlier $m-1$ admissible operations can be an operation of type (ad 5) which contains an operation (fad 4) which gives rise to the pivot $X$ of (ad 2) but from Lemma [51, Lemma 3.10] this case can be reduced to (ad $5^{*}$ ) which contains an operation (fad $\left.1^{*}\right)$. Moreover, from [46, Lemma 3.3] we know that, for a fixed $\mathbf{e} \in K_{0}(A)$, each ray and coray in $\Gamma$ contains at most one module $Z$ with $\operatorname{dim} Z=\mathbf{e}$. Therefore, we get that the number of new indecomposable modules with the same 
dimension vector is at most $t+1$ while after applying operations $\left(\operatorname{ad} 1^{*}\right)$ and $(\operatorname{ad} 2)$ or $\left(\operatorname{fad} 1^{*}\right)$ and $(\operatorname{ad} 2)$ the number of new vertices in ordinary quiver of $A$ increases by $t+2$. If the $m$ th admissible operation is of type ( $\left.\mathrm{ad} 2^{*}\right)$, then the proof is dual. If the $m$ th admissible operation is of type (ad 3), then in the sequence of earlier $m-1$ admissible operations, there must be at least one operation of type (ad $\left.1^{*}\right)$ or $\left(\operatorname{ad~} 5^{*}\right)$ which contains the operation $\left(\mathrm{fad} 1^{*}\right)$ which gives rise to the pivot $X$ of $(\operatorname{ad} 3)$ and to the modules $Y_{1}, Y_{2}, \ldots, Y_{t}$ in the support of $\operatorname{Hom}_{A}(X,-)$ restricted to the generalized multicoil containing $X$. The operations done after must not affect this support. Again, in general, in the sequence of earlier $m-1$ admissible operations can be an operation of type ( $\mathrm{ad} 5$ ) which contains an operation (fad 4) which gives rise to the pivot $X$ of (ad 3) but from [51, Lemma 3.10] this case can be reduced to $\left(\right.$ ad $\left.5^{*}\right)$ which contains an operation (fad $\left.1^{*}\right)$. Suppose that we had $r$ such consecutive operations of types $\left(\operatorname{ad} 1^{*}\right)$ or $\left(\operatorname{fad} 1^{*}\right)$, the first of which had $X_{t}$ as a pivot, and these admissible operations built up a branch $L$ in $A$ with points $a, a_{1}, a_{2}, \ldots, a_{t}$ in $Q_{A}$, so that $X_{t-1}$ and $Y_{t}$ are the indecomposable injective $A$-modules corresponding respectively to $a$ and $a_{1}$, and both $Y_{1}$ and $\tau_{A}^{-1} Y_{1}$ are coray modules in the generalized multicoil containing the (ad 3)-pivot $X$. Again, from [46, Lemma 3.3] we know that, for a fixed $\mathbf{e} \in K_{0}(A)$, each ray and coray in $\Gamma$ contains at most one module $Z$ with $\operatorname{dim} Z=\mathbf{e}$. Therefore, we get that the number of new indecomposable modules with the same dimension vector is at most $t+1$ while after applying $r$ consecutive operations of types $\left(\operatorname{ad} 1^{*}\right)$ and $(\operatorname{ad} 3)$ or $r$ consecutive operations of types (fad $\left.1^{*}\right)$ and (ad 3) the number of new vertices in ordinary quiver of $A$ increases by $t+2$. If the $m$ th admissible operation is of type ( $\left.\operatorname{ad} 3^{*}\right)$, then the proof is dual. If the $m$ th admissible operation is of type ( $\operatorname{ad} 4)$, then $A$ is the algebra obtained from another one, say $A^{\prime}$, by applying this admissible operation with pivot $X$ and the begin $Y_{1}$ of a finite sectional path $Y_{1} \rightarrow Y_{2} \rightarrow \cdots \rightarrow Y_{t}$. Note that this finite sectional path is the linearly oriented quiver of type $\mathbb{A}_{t}$ and its support algebra $\Lambda$ (given by the vertices corresponding to the simple composition factors of the modules $Y_{1}, Y_{2}, \ldots, Y_{t}$ ) is a tilted algebra of the path algebra $D$ of the linearly oriented quiver of type $\mathbb{A}_{t}$. From [65. (4.4)(2)] we know that $\Lambda$ is a bound quiver algebra given by a truncated branch in $x$, where $x$ corresponds to the unique projective-injective $D$-module. Moreover, the modules $Y_{1}, Y_{2}, \ldots, Y_{t}$ are directing in $\Gamma_{A^{\prime}}$. Again, from [46, Lemma 3.3] we know that, for a fixed $\mathbf{e} \in K_{0}(A)$, each ray and coray in $\Gamma$ contains at most one module $Z$ with $\operatorname{dim} Z=\mathbf{e}$. Therefore, we get that the number of new indecomposable modules with the same dimension vector is at most $t+r+1$ while after applying operations which give rise to the finite sectional path $Y_{1} \rightarrow Y_{2} \rightarrow \cdots \rightarrow Y_{t}$ and (ad 4) the number of new vertices in ordinary quiver of $A$ increases by $t+r+2$. If the $m$ th admissible operation is of type $\left(\mathrm{ad} 4^{*}\right)$, then the proof is dual. There remains to consider the case where the $m$ th admissible operation is of type $(\mathrm{ad} 5)$. Since in the definition of admissible operation (ad 5) we use the finite versions (fad 1), (fad 2), (fad 3), (fad 4) of the admissible operations ( $\operatorname{ad} 1),(\operatorname{ad} 2),(\operatorname{ad} 3),(\operatorname{ad} 4)$ and the admissible operation ( $\mathrm{ad} 4)$, we conclude that the lemma follows from the above considerations. If it is of type $\left(\operatorname{ad~} 5^{*}\right)$, then the proof is dual and this finishes the proof of the lemma.

The following proposition is essential for the proof of Theorem 8.3 
Proposition 8.2. Let A be a tame generalized multicoil algebra, $n$ the rank of $K_{0}(A)$, and $\mathbf{d}$ a nonnegative vector of $K_{0}(A)$. Then

(i) The number of isomorphism classes of discrete indecomposable A-modules $X$ with $\operatorname{dim} X=\mathbf{d}$ is bounded by $n+2$.

(ii) The number of isomorphism classes of indecomposable A-modules $X$ with $\operatorname{dim} X$ $=\mathbf{d}$ and $q_{A}(\operatorname{dim} X) \neq 0$ is bounded by $n-1$.

(iii) The number of isomorphism classes of indecomposable A-modules $X$ with $\operatorname{dim} X$ $=\mathbf{d}$ and $\chi_{A}(\operatorname{dim} X) \neq 0$ is bounded by $n-1$.

Proof. It is known that an indecomposable $A$-module $M$ in $\Gamma_{A}$ which does not lie on oriented cycle in $\Gamma_{A}$ is uniquely determined by $[M]$. Moreover, if $\mathscr{T}$ is a stable tube in $\Gamma_{A}$ then the support of $\mathscr{T}$ is a tame concealed or tubular convex subcategory of $A$. Hence, for any indecomposable $A$-module $X$ lying in a stable tubes of rank one, we have $q_{A}(\operatorname{dim} X)=\chi_{A}(\operatorname{dim} X)=0$. Let $\mathbf{d}$ be a nonnegative vector in $K_{0}(A)$ such that there exists a nondirecting, discrete indecomposable $A$-module $X$ with $\operatorname{dim} X=\mathbf{d}$. Then $X$ belongs to a generalized multicoil $\Gamma$ of $\Gamma_{A}$. Assume first that $\operatorname{Hom}_{A}(P, X) \neq 0$ for some indecomposable projective module in $\Gamma$. Then it follows from the proof of [46, Proposition 3.5] that any indecomposable $A$-module $Y$ with $\operatorname{dim} Y=\mathbf{d}$ also lies in $\Gamma$. Applying now Lemma 8.1 we conclude that the number of isomorphism classes of indecomposable $A$-modules $Z$ with $\operatorname{dim} Z=\operatorname{dim} X=\mathbf{d}$ is bounded by $n-1$. We get the same statement in the case when $\operatorname{Hom}_{A}(X, I) \neq 0$ for an indecomposable injective module $I$ in $\Gamma$. Note that different tame concealed algebras and different tubular algebras give modules in $A$ with different dimension vectors. Hence, it remains to consider the case when the support of $X$ is contained in a convex subcategory, say $C$, which is tame concealed or tubular. Then $X$ belongs to a $\mathbb{P}_{1}(K)$-family $\mathscr{T}=\left(\mathscr{T}_{\lambda}\right)_{\lambda \in \mathbb{P}_{1}(K)}$ of standard stable tubes of $\Gamma_{C}$. Moreover, if $Z$ is a indecomposable $A$-module with $\operatorname{dim} Z=\operatorname{dim} X=\mathbf{d}$ then $Z$ is a $C$-module and lies in one of the tubes $\mathscr{T}_{\lambda}$ (see [65] or [75]). Denote by $m$ the rank of $K_{0}(C)$, and by $r_{\lambda}$ the rank of the tube $\mathscr{T}_{\lambda}, \lambda \in \mathbb{P}_{1}(K)$. Then the following equality holds

$$
\sum_{\lambda \in \mathbb{P}_{1}(K)}\left(r_{\lambda}-1\right)=m-2
$$

(see [75]). Further, if $Y \in \mathscr{T}_{\lambda}$ and $Z \in \mathscr{T}_{\mu}$ are two nonisomorphic modules in $\mathscr{T}$ with $\operatorname{dim} Y=\operatorname{dim} Z$ then the quasi-length of $Y$ is divisible by $r_{\lambda}$ and the quasi-length of $Z$ is divisible by $r_{\mu}$. We note that then $q_{A}(\operatorname{dim} Y)=q_{C}(\operatorname{dim} Y)=\chi_{A}(\operatorname{dim} Y)=0$ and $q_{A}(\operatorname{dim} Z)=q_{C}(\operatorname{dim} Z)=\chi_{A}(\operatorname{dim} Z)=0$, since $g l \operatorname{dim} C \leq 2$. Now a simple inspection of tubular types of tame concealed and tubular algebras shows that, if $\lambda_{1}, \ldots, \lambda_{t}$ are all indices $\lambda \in \mathbb{P}_{1}(K)$ with $r_{\lambda} \neq 1$, then $r_{\lambda_{1}}+\ldots+r_{\lambda_{t}} \leq m+2 \leq n+2$. Therefore, the number of isomorphism classes of indecomposable $A$-modules $Z$ with $\operatorname{dim} Z=\operatorname{dim} X=\mathbf{d}$ is bounded by $n+2$.

Theorem 8.3. Let $A$ be a cycle-finite algebra. Then there is a positive integer $m$ such that, for each nonnegative vector $\mathbf{d} \in K_{0}(A)$, the number of isomorphism classes of discrete indecomposable A-modules of dimension vector $\mathbf{d}$ is bounded by $m$. 
Moreover, if $A$ is coherent, $n$ is the rank of $K_{0}(A)$, then the following statements hold:

(i) The number of isomorphism classes of discrete indecomposable A-modules $X$ with $\operatorname{dim} X=\mathbf{d}$ is bounded by $n+2$.

(ii) The number of isomorphism classes of indecomposable A-modules $X$ with $\operatorname{dim} X$ $=\mathbf{d}$ and $q_{A}(\operatorname{dim} X) \neq 0$ is bounded by $n-1$.

(iii) The number of isomorphism classes of indecomposable A-modules $X$ with $\operatorname{dim} X$ $=\mathbf{d}$ and $\chi_{A}(\operatorname{dim} X) \neq 0$ is bounded by $n-1$.

Proof. It follows from a result due to Ringel [65, (2.4)(8)] that, if $X, Y$ are modules in ind $A$ with $\operatorname{dim} X=\operatorname{dim} Y$ and $X$ is directing, then $X$ and $Y$ are isomorphic. Further, by Theorem 7.3 , there exist tame generalized multicoil quotient algebras $B_{1}, \ldots, B_{q}$ of $A$ such that all but finitely many isomorphism classes of nondirecting modules in ind $A$ belong to generalized multicoils of the Auslander-Reiten quivers $\Gamma_{B_{1}}, \ldots, \Gamma_{B_{q}}$ of $B_{1}, \ldots, B_{q}$. Moreover, it follows from the proof of Theorem 6.3 that, if $X$ and $Y$ are nondirecting discrete modules in ind $A$ with $\operatorname{dim} X=\operatorname{dim} Y$ lying in generalized multicoils of $\Gamma_{B_{1}}, \ldots, \Gamma_{B_{q}}$, then $X$ and $Y$ belong to the same generalized multicoil of $\Gamma_{B_{p}}$, for a fixed $p \in\{1, \ldots, q\}$. Then there is a positive integer $m$ such

that, for each nonnegative vector $\mathbf{d} \in K_{0}(A)$, the number of isomorphism classes of discrete indecomposable $A$-modules of dimension $\mathbf{d}$ is bounded by $m$. Finally, if $A$ is coherent, then all nondirecting modules in ind $A$ lie in generalized multicoils of $\Gamma_{B_{1}}, \ldots, \Gamma_{B_{q}}$, and the statements (i), (ii), (iii) are consequences of Proposition 8.2

\section{Homological properties of indecomposable modules}

Let $A$ be an algebra and $X$ be a nonprojective module in ind $A$. Then we have an almost split sequence

$$
0 \rightarrow \tau_{A} X \rightarrow \bigoplus_{i=1}^{s(X)} Y_{i} \rightarrow X \rightarrow 0
$$

in $\bmod A$ with $Y_{1}, \ldots, Y_{S(X)}$ indecomposable modules. We note that $\tau_{A}=D \operatorname{Tr}$, where $D=\operatorname{Hom}_{K}(-, K)$ is the standard duality on $\bmod A$ and the transpose $\operatorname{Tr} X$ of a module $X$ in ind $A$ is the cokernel of the homomorphism $\operatorname{Hom}_{A}(f, A)$ in $\bmod A^{\text {op }}$ associated to a minimal projective presentation $P_{1} \stackrel{f}{\longrightarrow} P_{0} \rightarrow X \rightarrow 0$ of $X$ in $\bmod A$. Hence $\tau_{A}$ is a homological operator, and $s(X)$ is a numerical homological invariant of a nonprojective module $X$ in ind $A$.

We also recall that for an indecomposable nonsimple projective-injective module $P$ in $\bmod A$ there is a canonical (up to isomorphism) almost split sequence having $P$ as a middle term, namely

$$
0 \rightarrow \operatorname{rad} P \rightarrow P \oplus \operatorname{rad} P / \operatorname{soc} P \rightarrow P / \operatorname{soc} P \rightarrow 0
$$


(see [1, Proposition IV.3.11]).

The following theorem has been proved by Bautista and Brenner in [12] (see also [44]).

Theorem 9.1. Let $A$ be an algebra of finite representation type and $X$ a nonprojective module in ind $A$. Then

(i) $s(X) \leq 4$.

(ii) If $s(X)=4$, then one of the indecomposable middle terms $Y_{i}$ of an almost split sequence for $X$ is projective-injective.

It has been conjectured by Brenner that, for any tame algebra $A$ and every nonprojective module $X$ in ind $A$, we have $s(X) \leq 5$. It is still an open problem. The following theorem proved in [60, Theorem 3] by the second named author and Takane confirms the Brenners's conjecture, and extends Theorem 9.1 to cycle-finite algebras.

Theorem 9.2. Let $A$ be a cycle-finite algebra and $X$ a nonprojective module in ind $A$. Then

(i) $s(X) \leq 5$.

(ii) If $s(X)=5$, then one of the indecomposable middle terms $Y_{i}$ of an almost split sequence for $X$ is projective-injective.

It has been proved by Ringel [65, (2.4)(8)] that, for any directing indecomposable module $X$ over an algebra $A$, we have $\operatorname{End}_{A}(X) \cong K$ and $\operatorname{Ext}_{A}^{r}(X, X)=0$ for $r \geq 0$. On the other hand, by [52, Theorem B], for a tame generalized multicoil algebra $A$ and an arbitrary module $X$ in ind $A$, we have $\operatorname{dim}_{K} \operatorname{Ext}_{A}^{1}(X, X) \leq \operatorname{dim}_{K} \operatorname{End}_{A}(X)$ and $\operatorname{Ext}_{A}^{r}(X, X)=0$ for $r \geq 2$. Hence, applying Theorems 6.3 and 6.5 (and Corollary 6.6) we obtain the following theorem.

Theorem 9.3. Let $A$ be a cycle-finite algebra. Then for all but finitely many isomorphism classes of module $X$ in ind $A$ we have $\operatorname{dim}_{K} \operatorname{Ext}_{A}^{1}(X, X) \leq \operatorname{dim}_{K} \operatorname{End}_{A}(X)$ and $\operatorname{Ext}_{A}^{r}(X, X)=0$ for $r \geq 2$.

We conclude from the above theorem that, for a cycle-finite algebra $A$ and all but finitely many isomorphism classes $X$ in ind $A$, the Euler form

$$
\chi_{A}([X])=\sum_{r=0}^{\infty}(-1)^{r} \operatorname{dim}_{K} \operatorname{Ext}_{A}^{r}(X, X)
$$

of $X$ is defined and is nonnegative. For $A$ a coherent cycle-finite algebra, it is the case for all modules $X$ in ind $A$.

It is known from [32, Theorem 2.3] and [63, Theorem 3.4] that if $A$ is a quasitilted algebra or a generalized double tilted algebra then, for all but finitely many isomorphism classes of modules $X$ in ind $A$, we have $\operatorname{pd}_{A} X \leq 1$ or $\operatorname{id}_{A} X \leq 1$. Moreover, it has been conjectured in [79] that the converse implication also holds.

We end this section by the following recent result by the third named author and Skowyrski [81] which confirms this conjecture for cycle-finite algebras. 
Theorem 9.4. Let $A$ be a cycle-finite algebra such that $\operatorname{pd}_{A} X \leq 1$ or $\operatorname{id}_{A} X \leq 1$ for all but finitely many isomorphism classes of modules in ind $A$. Then $A$ is a tame quasitilted algebra or a tame generalized double tilted algebra.

\section{Geometric properties of indecomposable modules}

The aim of this section is to present some results describing geometric properties of indecomposable modules over cycle-finite algebras.

Let $A$ be an algebra and $A \cong K Q / I$ its bound presentation. Then $I$ is an admissible ideal in the path algebra $K Q$ of $Q$ generated by a finite system of forms $\sum_{1 \leq j \leq t} \lambda_{j} \alpha_{m_{j}, j \ldots \alpha_{1, j}}$ (called $K$-linear relations), where $\lambda_{1}, \ldots, \lambda_{t}$ are elements of $K$ and $\alpha_{m_{j}, j}, \ldots, \alpha_{1, j}, 1 \leq j \leq t$, are paths of length $\geq 2$ in $Q$ having a common source and a common end. Denote by $Q_{0}$ the set of vertices of $Q$, by $Q_{1}$ the set of arrows of $Q$, and by $s, e: Q_{1} \rightarrow Q_{0}$ the maps which assign to each arrow $\alpha_{1}$ its source $s(\alpha)$ and its end $e(\alpha)$. The category $\bmod A$ of finite dimensional right $A$ modules is equivalent to the category $\operatorname{rep}_{K}(Q, I)$ of all finite dimensional representations $V=\left(V_{i}, \varphi_{\alpha}\right)_{i \in Q_{0}, \alpha \in Q_{1}}$ of $Q$, where $V_{i}, i \in Q_{0}$, are finite dimensional $K$-vector spaces and $\varphi_{\alpha}: V_{s(\alpha)} \rightarrow V_{e(\alpha)}, \alpha \in Q_{1}$, are $K$-linear maps satisfying the equations $\sum_{1 \leq j \leq t} \lambda_{j} \varphi_{\alpha_{m_{j}, j}} \ldots \varphi_{\alpha_{1, j}}=0$ for all $K$-linear relations $\sum_{1 \leq j \leq t} \lambda_{j} \alpha_{m_{j}, j} \ldots \alpha_{1, j} \in I$ (see [1]). Fix now a vector $\mathbf{d}=\left(d_{i}\right)_{i \in Q_{0}} \in K_{0}(A)=\mathbb{Z}^{Q_{0}}$ with nonnegative coordinates. Denote by $\bmod _{A}(\mathbf{d})$ the set of all representations $V=\left(V_{i}, \varphi_{\alpha}\right)$ in $\operatorname{rep}_{K}(Q, I)$ with $V_{i}=K^{d_{i}}$ for all $i \in Q_{0}$. A representation $V$ in $\bmod _{A}(\mathbf{d})$ is given by $d_{e(\alpha)} \times d_{s(\alpha)^{-}}$ matrices $V(\alpha)$ determining the maps $\varphi_{\alpha}: K^{s(\alpha)} \rightarrow K^{e(\alpha)}, \alpha \in Q_{1}$, in the canonical bases of $K^{d_{i}}, i \in Q_{0}$. Moreover, the matrices $V(\alpha), \alpha \in Q_{1}$, satisfy the relations

$$
\sum_{1 \leq j \leq t} \lambda_{j} V\left(\alpha_{m_{j}, j}\right) \ldots V\left(\alpha_{1, j}\right)=0
$$

for all $K$-linear relations $\sum_{1 \leq j \leq t} \lambda_{j} \alpha_{m_{j}, j} \ldots \alpha_{1, j} \in I$. Therefore, $\bmod _{A}(\mathbf{d})$ is a closed subset of $\mathbb{A}(\mathbf{d})=\prod_{\alpha \in Q_{1}} K^{d_{e(\alpha)} \times d_{s(\alpha)}}$ in the Zariski topology, and so $\bmod _{A}(\mathbf{d})$ is an affine variety. We note that $\bmod _{A}(\mathbf{d})$ is not necessarily irreducible. The affine (reductive) algebraic group $G(\mathbf{d})=\prod_{i \in Q_{0}} \mathrm{GL}_{d_{i}}(K)$ acts on the variety $\bmod _{A}(\mathbf{d})$ by conjugation

$$
(g V)(\alpha)=g_{e(\alpha)} V(\alpha) g_{s(\alpha)}^{-1}
$$

for $g=\left(g_{i}\right) \in G(\mathbf{d}), V \in \bmod _{A}(\mathbf{d}), \alpha \in Q_{1}$. We shall identify an $A$-module $V$ of dimension vector $\mathbf{d}$ with the corresponding point of the variety $\bmod _{A}(\mathbf{d})$. The $G(\mathbf{d})$ orbit $G(\mathbf{d}) M$ of a module $M$ in $\bmod _{A}(\mathbf{d})$ will be denoted by $\mathscr{O}(M)$. Observe that two modules $M$ and $N$ in $\bmod _{A}(\mathbf{d})$ are isomorphic if an only if $\mathscr{O}(M)=\mathscr{O}(N)$. For $M, N \in \bmod _{A}(\mathbf{d})$, we say that $N$ is a degeneration of $M$ if $N$ belongs to the Zariski closure $\overline{\mathscr{O}(M)}$ of $\mathscr{O}(M)$ in $\bmod _{A}(\mathbf{d})$, and we denote this fact by $M \leq_{\operatorname{deg}} N$. We note that $\leq_{\text {deg }}$ is a partial order in $\bmod _{A}(\mathbf{d})$. If $N \in \overline{\mathscr{O}(M)}$ implies $\mathscr{O}(N)=\mathscr{O}(M)$, then the orbit $\mathscr{O}(N)$ is said to be maximal. Clearly, an orbit in $\bmod _{A}(\mathbf{d})$ of maximal 
dimension is maximal, but the converse is not true in general. It is known that the union of all $G(\mathbf{d})$-orbits in $\bmod _{A}(\mathbf{d})$ of maximal dimension is an open subset of $\bmod _{A}(\mathbf{d})$, called an open sheet (see [38], [39]). We note also that for a module $M$ in $\bmod _{A}(\mathbf{d})$, we have $\operatorname{dim} \mathscr{O}(M)=\operatorname{dim} G(\mathbf{d})-\operatorname{dim}_{K} \operatorname{End}_{A}(M)($ see [38]). Given a module $M \in \bmod _{A}(\mathbf{d})$ we denote by $T_{M}\left(\bmod _{A}(\mathbf{d})\right)$ the tangent space of $\bmod _{A}(\mathbf{d})$ at $M$ and by $T_{M}(\mathscr{O}(M))$ the tangent space to $\mathscr{O}(M)$ at $M$. Then there is a canonical monomorphism of $K$-vector spaces

$$
T_{M}\left(\bmod _{A}(\mathbf{d})\right) / T_{M}(\mathscr{O}(M)) \hookrightarrow \operatorname{Ext}_{A}^{1}(M, M)
$$

(see [39. (2.7)]. In particular, if $\operatorname{Ext}_{A}^{1}(M, M)=0$ then $\overline{\mathscr{O}(M)}$ is an irreducible component of $\bmod _{A}(\mathbf{d})$ and $\mathscr{O}(M)$ is an open sheet of $\bmod _{A}(\mathbf{d})$. The local dimension $\operatorname{dim}_{M} \bmod _{A}(\mathbf{d})$ of $\bmod _{A}(\mathbf{d})$ is the maximal dimension of the irreducible components of $\bmod _{A}(\mathbf{d})$ containing $M$. We have $\operatorname{dim} T_{M}\left(\bmod _{A}(\mathbf{d})\right) \geq \operatorname{dim}_{M} \bmod _{A}(\mathbf{d})$. Further, $M \in \bmod _{A}(\mathbf{d})$ is said to be a nonsingular point of $\bmod _{A}(\mathbf{d})$ if $\operatorname{dim}_{M} \bmod _{A}(\mathbf{d})=$ $\operatorname{dim} T_{M}\left(\bmod _{A}(\mathbf{d})\right)$. If $M$ is a nonsingular point of $\bmod _{A}(\mathbf{d})$ then $M$ belongs to exactly one irreducible component of $\bmod _{A}(\mathbf{d})$ [66, (II.2.6)]. The nonsingular points of $\bmod _{A}(\mathbf{d})$ form an open nonempty subset. It is known that a module $M$ in $\bmod _{A}(\mathbf{d})$ is nonsingular provided $\operatorname{Ext}_{A}^{2}(M, M)=0$. A module variety $\bmod _{A}(\mathbf{d})$ is said to be a complete intersection provided the vanishing ideal of $\bmod _{A}(\mathbf{d})$ in the coordinate ring $K[\mathbb{A}(\mathbf{d})]$ of the affine space $\mathbb{A}(\mathbf{d})=\prod_{\alpha \in Q_{1}} K^{d_{e(\alpha)} \times d_{s(\alpha)}}$ is generated by $\operatorname{dim} \mathbb{A}(\mathbf{d})-\operatorname{dim} \bmod _{A}(\mathbf{d})$ polynomials. Finally, a module variety $\bmod _{A}(\mathbf{d})$ is said to be normal if the local ring $\mathscr{O}_{M}$ of any $\operatorname{module}_{M}$ in $\bmod _{A}(\mathbf{d})$ is integrally closed in its total quotient ring. It is known that if $\bmod _{A}(\mathbf{d})$ is normal then it is nonsingular in codimension one, that is, the set of singular points in $\bmod _{A}(\mathbf{d})$ is of codimension at most two (see [31, Chapter 11]). If $\bmod _{A}(\mathbf{d})$ is a complete intersection, then $\bmod _{A}(\mathbf{d})$ is normal if and only if $\bmod _{A}(\mathbf{d})$ is nonsingular in codimension one (consequence of Serre's normality criterion). In the study of the degeneration order on a module variety $\bmod _{A}(\mathbf{d})$ an important role is played by the following related partial orders. Let $M$ and $N$ be modules in a module variety. We define:

- $M \leq$ ext $N$ : $\Leftrightarrow$ there are modules $M_{i}, U_{i}, V_{i}$ and short exact sequences $0 \rightarrow U_{i} \rightarrow$ $M_{i} \rightarrow V_{i} \rightarrow 0$ in $\bmod A$ such that $M=M_{1}, M_{i+1}=U_{i} \oplus V_{i}, 1 \leq i \leq s$, and $N=M_{s+1}$ for some natural number $s$.

- $M \leq_{R} N$ : $\Leftrightarrow$ there exists in $\bmod A$ an exact sequence of the form $0 \rightarrow N \rightarrow M \oplus$ $Z \rightarrow Z \rightarrow 0$.

- $M \leq N$ : $\Leftrightarrow \operatorname{dim}_{K} \operatorname{Hom}_{A}(M, X) \leq \operatorname{dim}_{K} \operatorname{Hom}_{A}(N, X)$ for all modules $X$ in $\bmod A$.

It follows from the result due to Auslander [9] that $\leq$ is a partial order on the isomorphism classes of modules with the same dimension vector. Further, for modules $M$ and $N$ in $\bmod _{A}(\mathbf{d})$, we have $M \leq N$ if and only if $\operatorname{dim}_{K} \operatorname{Hom}_{A}(X, M) \leq$ $\operatorname{dim}_{K} \operatorname{Hom}_{A}(X, N)$ for all modules $X$ in $\bmod A$ by a result of Auslander and Reiten [10]. Moreover, by a result of Zwara [88], we have $M \leq_{R} N$ if and only if there exists in $\bmod A$ a short exact sequence of the form $0 \rightarrow Z^{\prime} \rightarrow Z^{\prime} \oplus M \rightarrow N \rightarrow 0$.

The following fundamental result of Zwara from [90] (see also [64] for the sufficiency part) gives an algebraic characterization of degenerations of modules. 
Theorem 10.1. Let $A$ be an algebra, $\mathbf{d}$ a vector in $K_{0}(A)$ with nonnegative coordinates, and $M, N$ modules in $\bmod _{A}(\mathbf{d})$. Then $M \leq_{\operatorname{deg}} N$ if and only if $M \leq_{R} N$.

In general, we have the following relations between the introduced orders. For modules $M$ and $N$ in the module variety $\bmod _{A}(\mathbf{d})$ the following implications hold

$$
M \leq_{\text {ext }} N \Longrightarrow M \leq_{\text {deg }} N \Longrightarrow M \leq N .
$$

Unfortunately, the reverse implications are not true in general, and it would be interesting to find out when there are true.

The following result of Zwara from [89] gives a combinatorial description of degenerations for modules over algebras of finite representation type.

Theorem 10.2. Let $A$ be an algebra of finite representation type, $\mathbf{d}$ a vector in $K_{0}(A)$ with nonnegative coordinates, and $M, N$ modules in $\bmod _{A}(\mathbf{d})$. Then $M \leq \operatorname{deg} N$ if and only if $M \leq N$.

We also exhibit the following results from [85] and [86] on degenerations of modules from the additive categories of generalized standard Auslander-Reiten components.

Theorem 10.3. Let $A$ be an algebra, $\mathscr{C}$ a generalized standard quasi-tube of $\Gamma_{A}$, and $M, N$ modules in $\operatorname{add}(\mathscr{C})$. Then $M \leq_{\operatorname{deg}} N$ if and only if $M \leq_{\mathrm{ext}} N$.

Theorem 10.4. Let $A$ be an algebra, $\mathscr{C}$ a generalized standard component of $\Gamma_{A}$, $N$ a module in $\operatorname{add}(\mathscr{C})$, and $M$ a module in $\bmod A$. If $M \leq_{\operatorname{deg}} N$ then $M$ belongs to $\operatorname{add}(\mathscr{C})$.

Theorem 10.5. Let $A$ be an algebra, $\mathscr{C}$ a generalized standard component of $\Gamma_{A}$, $M, N$ modules in $\operatorname{add}(\mathscr{C})$ with $\operatorname{dim} M=\operatorname{dim} N$. The following conditions are equivalent.

(i) $M \leq_{\text {deg }} N$.

(ii) There exists an exact sequence $0 \rightarrow N \rightarrow M \oplus Z \rightarrow Z \rightarrow 0$ in $\bmod A$ with $Z$ from $\operatorname{add}(\mathscr{C})$.

(iii) There exists an exact sequence $0 \rightarrow Z^{\prime} \rightarrow Z^{\prime} \oplus M \rightarrow N \rightarrow 0$ in $\bmod A$ with $Z^{\prime}$ from $\operatorname{add}(\mathscr{C})$.

(iv) $\operatorname{dim}_{K} \operatorname{Hom}_{A}(M, X) \leq \operatorname{dim}_{K} \operatorname{Hom}_{A}(N, X)$ for all modules $X$ in $\mathscr{C}$.

(v) $\operatorname{dim}_{K} \operatorname{Hom}_{A}(X, M) \leq \operatorname{dim}_{K} \operatorname{Hom}_{A}(X, N)$ for all modules $X$ in $\mathscr{C}$.

Let $A$ be an algebra and $M, N$ be nonisomorphic modules in ind $A$ with $\operatorname{dim} M=$ $\operatorname{dim} N$. Then $M \leq N$ forces the inequalities $\operatorname{dim}_{K} \operatorname{Hom}_{A}(M, M) \leq \operatorname{dim}_{K} \operatorname{Hom}_{A}(N, M)$ and $\operatorname{dim}_{K} \operatorname{Hom}_{A}(M, M) \leq \operatorname{dim}_{K} \operatorname{Hom}_{A}(M, N)$, and consequently we have a cycle $M \rightarrow N \rightarrow M$. Since $M \leq_{\operatorname{deg}} N$ implies $M \leq N$, we conclude that the directing modules in ind $A$ are never involved in proper degenerations of indecomposable modules. Observe also that, if $A$ is a cycle-finite algebra and $M<_{\operatorname{deg}} N$, then $M$ and $N$ belong to the same cyclic component of $\Gamma_{A}$. The degenerations of modules in the additive categories of generalized multicoils of Auslander-Reiten quivers of algebras were investigated in [47], [48], [83], [85]. Then, using Theorems 6.3 and 6.5, we obtain the following results. 
Theorem 10.6. Let $A$ be a cycle-finite algebra. Then there exists a positive integer $t$ such that for any sequence

$$
M_{r}<_{\operatorname{deg}} M_{r-1}<_{\operatorname{deg}} \ldots<_{\operatorname{deg}} M_{2}<_{\operatorname{deg}} M_{1}
$$

with $M_{1}, \ldots, M_{r}$ modules in ind $A$, the inequality $r \leq t$ holds.

Theorem 10.7. Let $A$ be a coherent cycle-finite algebra and $M, M^{\prime}, N$ be modules in $\bmod A$ such that $M<_{\operatorname{deg}} N, M^{\prime}<_{\operatorname{deg}} N$ and $N$ is indecomposable. Then $M \cong M^{\prime}$ and is indecomposable.

The geometry of directing modules over tame algebras has been described in [14]. In particular, we have the following consequence of [14, Theorems 1 and 2].

Theorem 10.8. Let $A$ be a tame algebra, $M$ a directing module in ind $A$, and $\mathbf{d}=$ $\operatorname{dim} M$. Then the following statements hold.

(i) $\bmod _{A}(\mathbf{d})$ is a complete intersection and has at most two irreducible components.

(ii) The maximal $G(\mathbf{d})$-orbits in $\bmod _{A}(\mathbf{d})$ consist of nonsingular modules.

(iii) $\mathscr{O}(M)$ is an open sheet of $\bmod _{A}(\mathbf{d})$.

(iv) All but finite number of $G(\mathbf{d})$-orbits in $\bmod _{A}(\mathbf{d})$ have codimension at least two.

(v) All $G(\mathbf{d})$-orbits of codimension one are contained in $\overline{\mathscr{O}(M)}$.

(vi) If $M$ is not projective-injective over $\operatorname{supp} M$ then $\bmod _{A}(\mathbf{d})=\mathscr{O}(M)$, is normal and a complete intersection.

It follows from Theorems 3.1 and 3.3 that the regular components of the AuslanderReiten quivers of cycle-finite algebras are stable tubes and their supports are tame concealed or tubular algebras. Then the following result from [16, Theorem 1] describes the geometry of modules from the additive categories of regular components of cycle-finite algebras.

Theorem 10.9. Let $A$ be a tame concealed or tubular algebra and $\mathbf{d}$ the dimension vector of a module in $\bmod A$ which is periodic with respect to the action of $\tau_{A}$. Then the affine variety $\bmod _{A}(\mathbf{d})$ is irreducible, normal, a complete intersection, $\operatorname{dim} \bmod _{A}(\mathbf{d})=\operatorname{dim} G(\mathbf{d})-q_{A}(\mathbf{d})$ and the maximal $G(\mathbf{d})$-orbits in $\bmod _{A}(\mathbf{d})$ form the open sheet consisting of nonsingular points. Moreover, a module $M$ in $\bmod _{A}(\mathbf{d})$ is a nonsingular point if and only if $\operatorname{Ext}_{A}^{2}(M, M)=0$.

We refer also to [15] for the geometry of indecomposable modules over tame quasi-tilted algebras, which give information on the geometry of indecomposable nondirecting modules of semiregular tubes of cycle-finite algebras (see Theorems 3.6 and 3.7.

We end this section with the following consequence of [52, Theorems A and B] and Theorem 6.3, extending [57, Theorem A] from strongly simply connected algebras of polynomial growth to coherent cycle-finite algebras.

Theorem 10.10. Let $A$ be a coherent cycle-finite algebra, $M$ a module in ind $A$ and $\mathbf{d}=\operatorname{dim} M$. Then the following statements hold.

(i) $M$ is a nonsingular point of $\bmod _{A}(\mathbf{d})$. 
(ii) $q_{A}(\mathbf{d}) \geq \chi_{A}(\mathbf{d})=\operatorname{dim}_{K} \operatorname{End}_{A}(M)-\operatorname{dim}_{K} \operatorname{Ext}_{A}^{1}(M, M) \geq 0$.

(iii) $\operatorname{dim}_{M} \bmod _{A}(\mathbf{d})=\operatorname{dim} G(\mathbf{d})-\chi_{A}(\mathbf{d})$.

We note that there are indecomposable modules $M$ over coherent cycle-finite $\operatorname{algebras} A$ with arbitrary large $\chi_{A}(\operatorname{dim} M)($ see $[58,(5.3)])$.

Acknowledgements The authors gratefully acknowledge supports from the grant Maestro of the National Science Center of Poland and the Centro de Investigación en Mathemáticas (CIMAT) Guanajuato in México.

\section{References}

1. Assem, I., Simson, D., Skowroński, A.: Elements of the Representation Theory of Associative Algebras 1: Techniques of Representation Theory. London Mathematical Society Student Texts, vol. 65, Cambridge University Press, Cambridge (2006)

2. Assem, I., Skowroński, A.: Iterated tilted algebras of type $\widetilde{\mathbb{A}}_{n}$. Math. Zeit. 195, 269-290 (1987)

3. Assem, I., Skowroński, A.: On some classes of simply connected algebras. Proc. London Math. Soc. 56, 417-450 (1988)

4. Assem, I., Skowroński, A.: Algebras with cycle-finite derived categories. Math. Ann. 280, 441-463 (1988)

5. Assem, I., Skowroński, A.: Minimal representation-infinite coil algebras. Manuscr. Math. 67, 305-331 (1990)

6. Assem, I., Skowroński, A.: Indecomposable modules over multicoil algebras. Math. Scand. 71, 31-61 (1992)

7. Assem, I., Skowroński, A.: Multicoil algebras. In: Representations of Algebras. CMS Conf. Proc. vol. 14, Amer. Math. Soc., pp. 29-68. Providence, RI (1993)

8. Assem, I., Skowroński, A., Tomé, B.: Coil enlargements of algebras. Tsukuba J. Math. 19, 453-479 (1995)

9. Auslander, M.: Representation theory of finite dimensional algebras. Contemp. Math. 13, 27 39 (1982)

10. Auslander, M., Reiten, I.: Modules determined by their composition factors. Illinois J. Math. 29, 280-301 (1985)

11. Auslander, M., Reiten, I., Smalø, S.O.: Representation Theory of Artin Algebras. Cambridge Studies in Advanced Mathematics, vol. 36, Cambridge University Press, Cambridge (1995)

12. Bautista, R., Brenner, S.: On the number of terms in the middle of an almost split sequence. In: Representations of algebras, Lecture Notes in Math., vol. 903, pp. 1-8. Springer, Berlin (1981)

13. Bautista, R., Gabriel, P., Roiter, A.V., Salmeron, L.: Representation-finite algebras and multiplicative bases. Invent. Math. 81, 217-285 (1985)

14. Bobiński, G., Skowroński, A.: Geometry of directing modules over tame algebras. J. Algebra 215, 603-643 (1999)

15. Bobiński, G., Skowroński, A.: Geometry of modules over tame quasi-tilted algebras. Colloq. Math. 79, 85-118 (1999)

16. Bobiński, G., Skowroński, A.: Geometry of periodic modules over tame concealed and tubular algebras. Algebras Represent. Theory 5, 187-200 (2002)

17. Bongartz, K.: Treue einfach zusammenhangende Algebren I. Comment. Math. Helvetici 57, 282-330 (1982)

18. Bongartz, K.: Algebras and quadratic forms. J. London Math. Soc. 28, 461-469 (1983) 
19. Bongartz, K.: Critical simply connected algebras. Manuscr. Math. 46, 117-136 (1984)

20. Bongartz, K.: A criterion for finite representation type. Math. Ann. 269, 1-12 (1984)

21. Bongartz, K.: Indecomposables are standard. Comment. Math. Helvetici 60, 400-410 (1985)

22. Bongartz, K., Gabriel, P.: Covering spaces in representation theory. Invent. Math. 65, 331-378 (1982)

23. Brenner, S., Butler, M.C.R.: Generalizations of the Bernstein-Gelfand-Ponomarev reflection functors. In: Representation Theory II, Lecture Notes in Math., vol. 832, pp. 103-169. Springer-Verlag, Berlin, Heidelberg, New York (1980)

24. Bretscher, O., Gabriel, P.: The standard form of representation-finite algebra. Bull. Soc. Math. France 111, 21-40 (1983)

25. Brüstle, T., de la Peña, J.A., Skowroński, A.: Tame algebras and Tits quadratic form. Advances Math. 226, 887-951 (2011)

26. Crawley-Boevey, W.: On tame algebras and bocses. Proc. London Math. Soc. 56, 451-483 (1988)

27. Crawley-Boevey, W.: Tame algebras and generic modules. Proc. London Math. Soc. 63, 241$265(1991)$

28. D'Este, G., Ringel, C.M.: Coherent tubes. J. Algebra 87, 150-201 (1984)

29. Dräxler, P.: Aufrichtige gerichtete Ausnahmealgebren. Bayreuter Math. Schriften 29 (1989).

30. Drozd, Y.A.: Tame and wild matrix problems. In: Representation Theory II, Lecture Notes in Math., vol. 832, pp. 242-258. Springer-Verlag, Berlin, Heidelberg, New York (1980)

31. Eisenbud, D.: Commutative Algebra with a View toward Algebraic Geometry. Graduate Texts in Math., vol. 150. Springer-Verlag, New York, Berlin (1996)

32. Happel, D., Reiten, I., Smalø, S.O.: Tilting in abelian categories and quasitilted algebras. Memoirs Amer. Math. Soc. 120, no. 575 (1996)

33. Happel, D., Ringel, C.M.: Tilted algebras. Trans. Amer. Math. Soc. 274, 399-443 (1982)

34. Happel, D., Vossieck, D.: Minimal algebras of infinite representation type with preprojective component. Manuscr. Math. 42, 221-243 (1983)

35. Hoshino, M.: Modules without self-extensions and Nakayama's conjecture. Archiv. Math. (Basel) 43, 493-500 (1984)

36. Jaworska, A., Malicki P., Skowroński, A.: On Auslander-Reiten components of algebras without external short paths. J. London Math. Soc. 85, 245-268 (2012)

37. Kerner, O.: Tilting wild algebras. J. London Math. Soc. 39, 29-47 (1989)

38. Kraft, H.: Geometric methods in representation theory. In: Representations of Algebras, Lecture Notes in Math., vol. 944, pp. 180-258. Springer-Verlag, Berlin, New York (1982)

39. Kraft, H.: Geometrische Methoden in der Invariantentheorie. Aspects of Mathematics D1, Friedr. Vieweg and Sohn, Braunschweig (1984)

40. Lenzing H., Skowroński, A.: Quasi-tilted algebras of canonical type. Colloq. Math. 71, 161181 (1996)

41. Liu, S.: Degrees of irreducible maps and the shapes of the Auslander-Reiten quivers. J. London Math. Soc. 45, 32-54 (1992)

42. Liu, S.: Semi-stable components of an Auslander-Reiten quiver. J. London Math. Soc. 47, 405-416 (1993)

43. Liu, S.: Tilted algebras and generalized standard Auslander-Reiten components. Archiv. Math. (Basel) 61, 12-19 (1993)

44. Liu, S.: Almost split sequences for non-regular modules. Fund. Math. 143, 183-190 (1993)

45. Malicki, P.: Generalized coil enlargements of algebras. Colloq. Math. 76, 57-83 (1998)

46. Malicki, P.: On the composition factors of indecomposable modules in almost cyclic coherent Auslander-Reiten components. J. Pure Appl. Algebra 207, 469-490 (2006)

47. Malicki, P.: Degenerations in the module varieties of almost cyclic coherent Auslander-Reiten components. Colloq. Math. 114, 253-276 (2009) 
48. Malicki, P.: Degenerations for indecomposable modules in almost cyclic coherent AuslanderReiten components, J. Pure Appl. Algebra 214, 1701-1717 (2010)

49. Malicki, P., de la Peña, J.A., Skowroński, A.: Cyclic components of the Auslander-Reiten quivers of cycle-finite algebras. Preprint, Toruń (2012)

50. Malicki, P., Skowroński, A.: Almost cyclic coherent components of an Auslander-Reiten quiver. J. Algebra 229, 695-749 (2000)

51. Malicki, P., Skowroński, A.: Algebras with separating almost cyclic coherent AuslanderReiten components. J. Algebra 291, 208-237 (2005)

52. Malicki, P., Skowroński, A.: On the additive categories of generalized standard almost cyclic coherent Auslander-Reiten components. J. Algebra 316, 133-146 (2007)

53. Peng, L.G., Xiao, J.: On the number of $D$ Tr-orbits containing directing modules. Proc. Amer. Math. Soc. 118, 753-756 (1993)

54. de la Peña, J.A.: On the dimension of the module-varieties of tame and wild algebras. Comm. Algebra 19, 1795-1807 (1991)

55. de la Peña, J.A.: Tame algebras with sincere directing modules. J. Algebra 161, 171-185 (1993)

56. de la Peña, J.A.: The families of two-parametric tame algebras with sincere directing modules. In: Representations of Algebras, CMS Conf. Proc. vol. 14, Amer. Math. Soc., pp. 361-392. Providence, RI (1993)

57. de la Peña, J.A., Skowroński, A.: Geometric and homological characterizations of polynomial growth strongly simply connected algebras. Invent. Math. 126, 287-296 (1996)

58. de la Peña, J.A., Skowroński, A.: The Tits and Euler forms of a tame algebra. Math. Ann. 315, 37-59 (1999)

59. de la Peña, J.A., Skowroński, A.: Algebras with cycle-finite Galois coverings. Trans. Amer. Math. Soc. 363, 4309-4336 (2011)

60. de la Peña, J.A., Takane, M.: On the number of terms in the middle of almost split sequences over tame algebras. Trans. Amer. Math. Soc. 351, 3857-3868 (1999)

61. de la Peña, J.A., Tomé, B.: Iterated tubular algebras. J. Pure Appl. Algebra 64, 303-314 (1990)

62. Reiten, I., Skowroński, A.: Characterizations of algebras with small homological dimensions. Advances Math. 179, 122-154 (2003)

63. Reiten, I., Skowroński, A.: Generalized double tilted algebras. J. Math. Soc. Japan 56, 269$288(2004)$

64. Riedtmann, C.: Degenerations for representations of quivers with relations. Ann. Sci. Ecole Normal Sup. 4, 275-301 (1986)

65. Ringel, C.M.: Tame Algebras and Integral Quadratic Forms. Lecture Notes in Math., vol. 1099, Springer-Verlag, Berlin, Heidelberg, New York (1984)

66. Shafarevich, I,R.: Basic algebraic geometry. Graduate Texts in Math. 213. Springer-Verlag (1977)

67. Simson, D., Skowroński, A.: Elements of the Representation Theory of Associative Algebras 2: Tubes and Concealed Algebras of Euclidean Type. London Mathematical Society Student Texts, vol. 71, Cambridge University Press, Cambridge (2007)

68. Simson, D., Skowroński, A.: Elements of the Representation Theory of Associative Algebras 3: Representation-Infinite Tilted Algebras. London Mathematical Society Student Texts, vol. 72, Cambridge University Press, Cambridge (2007)

69. Skowroński, A.: Selfinjective algebras of polynomial growth. Math. Ann. 285, 177-199 (1989)

70. Skowroński, A.: Algebras of polynomial growth. In: Topics in Algebra, vol. 26, Part 1, pp. 535-568. PWN Warsaw (1990)

71. Skowroński, A.: Simply connected algebras and Hochschild cohomologies. In: Representations of Algebras, CMS Conf. Proc. vol. 14, Amer. Math. Soc., pp. 431-447. Providence, RI (1993) 
72. Skowroński, A.: Generalized standard Auslander-Reiten components without oriented cycles. Osaka J. Math. 30, 515-527 (1993)

73. Skowroński, A.: Generalized standard Auslander-Reiten components. J. Math. Soc. Japan 46, 517-543 (1994)

74. Skowroński, A.: Regular Auslander-Reiten components containing directing modules. Proc. Amer. Math. Soc. 120, 19-26 (1994)

75. Skowroński, A.: On the composition factors of periodic modules. J. London Math. Soc. 46, 477-492 (1994)

76. Skowroński, A.: Cycle-finite algebras. J. Pure Appl. Algebra 103, 105-116 (1995)

77. Skowroński, A.: Simply connected algebras of polynomial growth. Compositio Math. 109, 99-133 (1997)

78. Skowroński, A.: Tame quasi-tilted algebras. J. Algebra 203, 470-490 (1998)

79. Skowroński, A.: On artin algebras with almost all indecomposable modules of projective or injective dimension at most one. Cent. Eur. J. Math. 1, 108-122 (2003)

80. Skowroński, A.: Selfinjective algebras: finite and tame type. In: Trends in Representation Theory of Algebras and Related Topics, Contemporary Math., vol. 406, Amer. Math. Soc., pp. 169-238. Providence, RI (2006)

81. Skowroński, A., Skowyrski, A.: Cycle-finite algebras with almost all indecomposable modules of projective or injective dimension at most one. Preprint, Torun (2012)

82. Skowroński, A., Yamagata, K.: Frobenius Algebras I. Basic Representation Theory. European Mathematical Society Textbooks in Mathematics, European Mathematical Society Publishing House, Zürich (2011)

83. Skowroński, A., Zwara, G.: On degenerations of modules with nondirecting indecomposable summands. Canad. J. Math. 48, 1091-1120 (1996)

84. Skowroński, A., Zwara, G.: On the numbers of discrete indecomposable modules over tame algebras. Colloq. Math. 73, 93-114 (1997)

85. Skowroński, A., Zwara, G.: Degenerations for indecomposable modules and tame algebras. Ann. Scient. Éc. Norm. Sup. 31, 153-180 (1998)

86. Skowroński, A., Zwara, G.: Degeneration-like orders on the additive categories of generalized standard Auslander-Reiten components. Arch. Math. 74, 11-21 (2000)

87. Zhang, Y.: The structure of stable components. Canad. J. Math. 43, 652-672 (1991)

88. Zwara, G.: A degeneration-like order for modules. Arch. Math. (Basel) 71, 437-444 (1998)

89. Zwara, G.: Degenerations for modules over representation-finite algebras. Proc. Amer. Math. Soc. 127, 1313-1322 (1999)

90. Zwara, G.: Degenerations of finite dimensional modules are given by extensions. Compositio Math. 121, 205-218 (2000) 